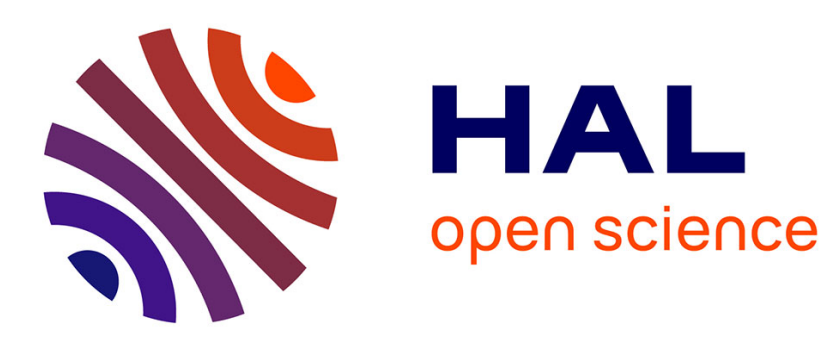

\title{
Weighted Mourre's commutator theory, application to Schrödinger operators with oscillating potential
}

\author{
Sylvain Golenia, Thierry Jecko
}

\section{To cite this version:}

Sylvain Golenia, Thierry Jecko. Weighted Mourre's commutator theory, application to Schrödinger operators with oscillating potential. Journal of Operator Theory, 2013, 1, J. Operator Theory 70 (2013), no. 1, 109-144. 10.7900/jot.2011may06.1922 . hal-00542694v3

\section{HAL Id: hal-00542694 \\ https://hal.science/hal-00542694v3}

Submitted on 1 Jun 2012

HAL is a multi-disciplinary open access archive for the deposit and dissemination of scientific research documents, whether they are published or not. The documents may come from teaching and research institutions in France or abroad, or from public or private research centers.
L'archive ouverte pluridisciplinaire HAL, est destinée au dépôt et à la diffusion de documents scientifiques de niveau recherche, publiés ou non, émanant des établissements d'enseignement et de recherche français ou étrangers, des laboratoires publics ou privés. 


\title{
WEIGHTED MOURRE'S COMMUTATOR THEORY, APPLICATION TO SCHRÖDINGER OPERATORS WITH OSCILLATING POTENTIAL
}

\author{
GOLÉNIA, SYLVAIN AND JECKO, THIERRY
}

\begin{abstract}
We present a variant of Mourre's commutator theory. We apply it to prove the limiting absorption principle for Schrödinger operators with a perturbed Wigner-Von Neumann potential at suitable energies. To our knowledge, this result is new since we allow a long range perturbation of the Wigner-Von Neumann potential. Furthermore, we can show that the usual Mourre theory, based on differential inequalities and on the generator of dilation, cannot apply to the mentioned Schrödinger operators.
\end{abstract}

\section{Contents}

1. Introduction.

2. Basic notions and notation.

2.1. Regularity.

2.2. Pseudodifferential calculus.

3. Weighted Mourre theory. 9

3.1. Reduced resolvent. 9

3.2. Special sequences. 9

3.3. Energy estimates. 11

3.4. Application. 12

4. Perturbed Wigner-Von Neumann potentials. 13

4.1. Definitions and regularity. 13

4.2. Energy localization of oscillations. 14

4.3. Usual Mourre estimate. $\quad 15$

$\begin{array}{ll}\text { 4.4. Weighted Mourre estimate. } & 17\end{array}$

5. Usual Mourre theory. 20

Appendix A. Oscillating terms. 24

Date: June 1, 2012.

Key words and phrases. Mourre's commutator theory, Mourre estimate, limiting absorption principle, continuous spectrum, Schrödinger operators, Wigner-Von Neumann potential. 
Appendix B. Functional calculus for pseudodifferential operators.

Appendix C. An interpolation's argument. 28

Appendix D. A simpler argument in dimension $d=1 . \quad 29$

References

\section{INTRODUCTION.}

Since its introduction in 1980 (cf., [Mo]), many papers have shown the power of Mourre's commutator theory to study the point and continuous spectra of a quite wide class of self-adjoint operators. Among others, we refer to [BFS, BCHM, CGH, DJ, FH, GGM1, GGo, HuS, JMP, Sa] and to the book [ABG]. One can also find parameter dependent versions of the theory (a semi-classical one for instance) in [RoT, Wa, WZ]. Recently it has been extended to (non self-adjoint) dissipative operators (cf., [BG, Roy]).

In [GJ], we introduced a new approach of Mourre's commutator theory, which is strongly inspired by results in semi-classical analysis (cf., [Bu, CJ, Je1, Je2]). In [Gé], C. Gérard showed that it can be followed using traditional "energy estimates". This approach furnishes an alternative way to develop the original Mourre Theory and do not use differential inequalities.

The aim of the present paper is to present a new theory, which shares common goals with Mourre's commutator theory but relies on different assumptions. It is inspired by the approach in [GJ] and in [Gé]. It is actually new since we can produce an example for which it applies while the strongest versions of Mourre's commutator theory (cf., [ABG, Sa]) with (variants of) the generator of dilation as conjugate operator cannot be applied to it.

Our example is a perturbation of a Schrödinger operator with a Wigner-Von Neumann potential. Furthermore we can allow a long range perturbation which is not covered by previous results in [DMR, ReT1, ReT2]. A similar situation is considered in $[\mathrm{MU}]$ but at different energies.

Let us now briefly recall Mourre's commutator theory and present our results. We need some notation and basic notions (see Section 2 for details). We consider two self-adjoint (unbounded) operators $H$ and $A$ acting in some complex Hilbert space $\mathscr{H}$. Let $\|\cdot\|$ denote the norm of bounded operators on $\mathscr{H}$. With the help of $A$, we study spectral properties of $H$, the spectrum $\sigma(H)$ of which is included in $\mathbb{R}$. Let $\mathcal{I}, \mathcal{J}$ be open intervals of $\mathbb{R}$. Given $k \in \mathbb{N}$, we say that $H \in \mathcal{C}_{\mathcal{J}}^{k}(A)$ if for all $\chi \in$ $\mathcal{C}_{c}^{\infty}(\mathbb{R})$ with support in $\mathcal{J}$, for all $f \in \mathscr{H}$, the map $\mathbb{R} \ni t \mapsto e^{i t A} \chi(H) e^{-i t A} f \in \mathscr{H}$ has the usual $\mathcal{C}^{k}$ regularity. Denote by $E_{\mathcal{I}}(H)$ the spectral measure of $H$ above $\mathcal{I}$. We say that the Mourre estimate holds true for $H$ on $\mathcal{I}$ if there exist $c>0$ and a compact operator $K$ such that

$$
E_{\mathcal{I}}(H)[H, i A] E_{\mathcal{I}}(H) \geq E_{\mathcal{I}}(H)(c+K) E_{\mathcal{I}}(H),
$$

in the form sense on $(\mathcal{D}(A) \cap \mathcal{D}(H)) \times(\mathcal{D}(A) \cap \mathcal{D}(H))$. In general, the 1.h.s. of (1.1) does not extend, as a form, on $\mathscr{H} \times \mathscr{H}$ but it is the case if $H \in \mathcal{C}_{\mathcal{J}}^{1}(A)$ and $\mathcal{I} \subset \mathcal{J}$ (cf., $[\mathrm{Sa}, \mathrm{GJ}]$ ). We say that the strict Mourre estimate holds true 
if the Mourre estimate (1.1) holds true with $K=0$. In the first case (resp. the second case), it turns out that the point spectrum of $H$ is finite (resp. empty) in compact sub-intervals $\mathcal{I}^{\prime}$ of $\mathcal{I}$ if $H \in \mathcal{C}_{\mathcal{J}}^{k}(A)$ and $\mathcal{I} \subset \mathcal{J}$. The main aim of Mourre's commutator theory is to show, when the strict Mourre estimate holds true for $H$ on $\mathcal{I}$, the following limiting absorption principle (LAP) on compact sub-intervals $\mathcal{I}^{\prime}$ of $\mathcal{I}$. Given such a $\mathcal{I}^{\prime}$ and $s>1 / 2$, we say that the LAP, respectively to the triplet $\left(\mathcal{I}^{\prime}, s, A\right)$, holds true for $H$ if

$$
\sup _{\operatorname{Re} z \in \mathcal{I}^{\prime}, \operatorname{Im} z \neq 0}\left\|\langle A\rangle^{-s}(H-z)^{-1}\langle A\rangle^{-s}\right\|<\infty,
$$

where $\langle t\rangle=\left(1+|t|^{2}\right)^{1 / 2}$. In that case, it turns out that the spectrum of $H$ is purely absolutely continuous in $\mathcal{I}^{\prime}$ (cf., Theorem XIII.20 in [RS4]). Notice that (1.2) holds true for $s=0$ if and only if $\mathcal{I}^{\prime} \cap \sigma(H)=\emptyset$.

In $[\mathrm{ABG}, \mathrm{Sa}]$, such LAPs are derived under a slightly stronger regularity assumption than $H \in \mathcal{C}_{\mathcal{J}}^{1}(A)$ with $\mathcal{I} \subset \mathcal{J}$. Actually, stronger results are proved. In particular, in the norm topology of bounded operators, one can defined the boundary values of the resolvent:

$$
\mathcal{I}^{\prime} \ni \lambda \mapsto \lim _{\varepsilon \rightarrow 0^{ \pm}}\langle A\rangle^{-s}(H-\lambda-i \varepsilon)^{-1}\langle A\rangle^{-s}
$$

and show some Hölder continuity for them.

Implicitly in [GJ] and explicitly in [Gé], one can derive, using $H \in \mathcal{C}_{\mathcal{J}}^{2}(A)$ with $\mathcal{I} \subset \mathcal{J}$, the LAP (1.2) on compact sub-intervals $\mathcal{I}^{\prime}$ of $\mathcal{I}$ from the Mourre estimate (1.1) with $K=0$ via a strict, weighted Mourre estimate:

$$
E_{\mathcal{I}}(H)[H, i \varphi(A)] E_{\mathcal{I}}(H) \geq c_{1} E_{\mathcal{I}}(H)\langle A\rangle^{-1-\varepsilon} E_{\mathcal{I}}(H),
$$

where $\varepsilon=2 s-1>0$ and $\varphi$ is some appropriate non-negative, bounded, smooth function on $\mathbb{R}$. Note that the l.h.s. of (1.4) is a well defined form on $\mathscr{H} \times \mathscr{H}$. It seems that the use of such kind of inequality to derive resolvent estimates appears in [Je1] for the first time.

Our new idea is to take the strict, weighted Mourre estimate (1.4) as starting point, instead of the strict Mourre estimate. This costs actually less regularity of $H$ w.r.t. A. Precisely, we show

Theorem 1.1. Let $\mathcal{I}$ be a bounded, open interval of $\mathbb{R}$ and assume that $H \in \mathcal{C}_{\mathcal{I}}^{1}(A)$. Assume that, for some $\varepsilon_{0}>0$, for any $\varepsilon \in\left(0 ; \varepsilon_{0}\right]$, there exists some real borelian bounded function $\varphi$ such that the strict, weighted Mourre estimate, i.e. (1.4), holds true. Then, for any $s>1 / 2$ and for any closed sub-interval $\mathcal{I}^{\prime}$ of $\mathcal{I}$, the LAP (1.2) for $H$ respectively to $\left(\mathcal{I}^{\prime}, s, A\right)$ holds true.

Remark 1.2. Notice that the LAP (1.2) for $H$ respectively to $\left(\mathcal{I}^{\prime}, s, A\right)$ implies the LAP (1.2) for $H$ respectively to $\left(\mathcal{I}^{\prime}, s^{\prime}, A\right)$, for any $s^{\prime} \geq s$. Therefore, it is enough to prove Theorem 1.1 for $s$ close to $1 / 2$.

Remark 1.3. Using Gérard's energy method in [Gé], we can upper bound the size of the l.h.s. of (1.2) in terms of the constant $c_{1}$ appearing in (1.4). See Corollary 3.7.

Actually Theorem 1.1 will follow from the more general result obtained in Theorem 3.4. The new theory that we present here and that we call "weighted Mourre theory" is essentially a part of the variant of the Mourre theory in [Gé, GJ]. As such, it is simpler than the usual Mourre theory (it does not use differential inequalities). However, we do not know if such approach gives continuity results on 
the boundary values of the resolvent (1.3). We shall give two (almost equivalent) ways to view the new theory (cf., Subsections 3.2 and 3.3).

As announced above, we want to derive the LAP (1.2) (for some $A$ ) on carefully chosen intervals $\mathcal{I}^{\prime}$ for a certain class of Schrödinger operators. Let $d \in \mathbb{N}^{*}$ and let $H_{0}$ be the self-adjoint realization of the Laplacian $-\Delta_{x}$ in $\mathrm{L}^{2}\left(\mathbb{R}_{x}^{d}\right)$. Given $q \in \mathbb{R}^{*}$ and $k>0$, the function $W: \mathbb{R}^{d} \longrightarrow \mathbb{R}$ defined by $W(x)=q(\sin k|x|) /|x|$ is called the Wigner-Von Neumann potential. We consider another real valued function $V$ satisfying some long range condition (see Section 4 for details) such that the operator $H_{1}:=H_{0}+W+V$ is self-adjoint on the domain of $H_{0}$. This is the Schrödinger operator with a perturbed Wigner-Von Neumann potential that we consider. It is well known that its essential spectrum is $[0 ;+\infty[$. Now we look for an interval $\left.\mathcal{I}^{\prime} \subset\right] 0 ;+\infty[$ on which we can get the $\operatorname{LAP}(1.2)$. As operator $A$, it is natural to choose the generator of dilation $A_{1}$, the self-adjoint realization of $\left(x \cdot \nabla_{x}+\nabla_{x} \cdot x\right) /(2 i)$ in $\mathrm{L}^{2}\left(\mathbb{R}_{x}^{d}\right)$. Indeed, when $W$ is absent, such LAPs have been derived. As mentioned above, the pure point spectrum $\sigma_{p p}\left(H_{1}\right)$ of $H_{1}$ has to be empty in $\mathcal{I}^{\prime}$.

There are many papers on the absence of positive eigenvalue for Schrödinger operators: see [Ka, Si, Ag, FHHH2, FH, IJ, RS4, CFKS]. They do not apply to the present situation because of the behaviour of the Wigner-Von Neumann $W$. One can even show that $k^{2} / 4$ is actually an eigenvalue of $H_{1}$ for a well chosen, radial, short range potential $V$ (cf., [RS4] p. 223 and [BD]).

In dimension $d=1$, the eigenvalue at $k^{2} / 4$ is preserved under suitable perturbation (see $[\mathrm{CHM}]$ ). Furthermore it is proved in [FH, FHHH1] that, if $|q|<k$, the usual Mourre estimate (1.1) holds true on compact intervals $\mathcal{I} \subset] 0 ;+\infty[$ and there is no eigenvalue in $] 0 ;+\infty[$, and otherwise that, on compact $\mathcal{I} \subset] 0 ;+\infty\left[\backslash\left\{k^{2} / 4\right\}\right.$, no eigenvalue is present and the usual Mourre estimate (1.1) holds true. Actually if $k^{2} / 4$ is an eigenvalue of $H_{1}$ then the usual Mourre estimates cannot hold true on a compact neighbourhood of $k^{2} / 4$, with the generator of dilation as conjugate operator. This follows from the arguments of the proof of Corollary 2.6 in $[\mathrm{FH}]$. Thus the eigenvalue $k^{2} / 4$ is a threshold.

We focus on compact intervals $\mathcal{I}$ satisfying $\left\{0, k^{2} / 4\right\} \cap \mathcal{I}=\emptyset$ and, when $d>1$, $\mathcal{I} \subset] 0 ; k^{2} / 4$. Using pseudodifferential calculus and recycling arguments from $[\mathrm{FH}]$, we prove the usual Mourre estimate (1.1) on such $\mathcal{I}$, the operator $A$ being $A_{1}$, yielding the finiteness of the pure point spectrum $\sigma_{p p}\left(H_{1}\right)$ in $\mathcal{I}$. Then, in Theorem 4.15, we derive a strict, weighted Mourre estimate (1.4) and show that Theorem 1.1 applies, leading to the LAP (1.2) for $H_{1}$. For short range perturbation $V$, we partially recover results from [DMR, ReT1, ReT2] but, in contrast to these papers, we are able to treat a long range perturbation $V$. We mention that in [MU], for high enough energies, one proves a LAP for long-range perturbations of a larger class of oscillating potentials. In this situation, the Laplacian is a priori "stronger" than the potential, in contrast to the present case.

As already mentioned, the LAP for $H_{1}$ implies the absence of singular spectrum over $\mathcal{I}$. This result seems to be new, even in dimension 1 . Concerning this question for potentials, which are "decaying at most like $1 / x$ at infinity", we refer to [Ki, Re] and references therein.

Finally we show that $H_{1}$ does not have the required regularity w.r.t. (variants of) $A_{1}$ to apply the usual Mourre theory from [ABG, GGM1, Sa]. For the same reason, the derivation of the strict, weighted Mourre estimate (1.4) for $H_{1}$ from the corresponding strict Mourre estimate, i.e. (1.1) with $K=0$, along the lines in [Gé], is 
not allowed. If one removes the oscillating potential $W$ and keeps our assumptions on $V=V_{\mathrm{lr}}+V_{\mathrm{sr}}$ (see Assumption 4.13), the situation is well-known. However, to obtain a LAP with the traditionnal Mourre theory, one needs some strong and quite involved versions as in [ABG, Sa]. In particular, the results in [Mo, Gé] do not apply in this case, while our arguments here gives the LAP (1.2) on any compact interval $\mathcal{I} \subset] 0 ;+\infty[$ (cf., Remarks 4.5 and 4.16 below).

We did not optimize our study of Schrödinger operators with oscillating potential. We believe that we can handle more general perturbations. Because of a difficulty explained in Remark 4.5, we did not consider intervals $\mathcal{I}$ above $k^{2} / 4$ for $d>1$. However we believe that a variant of the present theory is applicable in this case. We think that a general study of long range perturbations of the Schrödinger operator with Wigner-Von Neumann potential is interesting in itself and hope to develop it in a forthcoming paper.

The paper is organized as follows. In Section 2, we introduce some notation and basic but important notions. In Section 3, we show a stronger version of Theorem 1.1, namely Theorem 3.4. In Section 4, we study Schrödinger operators with perturbed Wigner-Von Neumann potentials. In Subsection 4.3, we derive usual Mourre estimates below the "threshold" $k^{2} / 4$. In Subsection 4.4, we essentially apply Theorem 1.1 to Schrödinger operators. In Section 5, we prove that they cannot be treated by the usual Mourre theory in $[\mathrm{ABG}, \mathrm{Sa}]$. In Appendix A, we prove a key pseudodifferential result to control the behaviour of the Wigner-Von Neumann potential (extending a result by $[\mathrm{FH}]$ in dimension one). In Appendix $\mathrm{B}$, we review functional calculus for pseudodifferential operators (cf., [Bo1]). In Appendix C, we establish the boundedness of some operator using interpolation. Finally, in Appendix $\mathrm{D}$, we present, in dimension one, a simpler proof of Lemma 5.5, this lemma being used to show that the regularity assumption of the usual Mourre theory is not satisfied by the Schrödinger operators studied here.

Acknowledgement: The authors thank Jean-Michel Bony, Vladimir Georgescu, Ira Herbst, Andreas Knauf, Jacob Schach Møller, Nicolas Lerner, Karel PravdaStarov, and Erik Stibsted for fruitful discussions.

The authors apologize to Jacob Schach Møller for not citing his paper [Mø] in their previous work [GJ] when they proved Proposition 2.6 and 2.7 below. They did not realize the presence of the corresponding result in $[\mathrm{M} \varnothing]$.

\section{BASIC NOTIONS AND NOTATION.}

In this section, we introduce some notation and recall known results. For details, we refer to $[\mathrm{ABG}, \mathrm{DG}, \mathrm{GJ}, \mathrm{Sa}]$ on regularity and to [Hö3, Bo1, Bo2, BC, Le] on pseudodifferential calculus.

2.1. Regularity. For an interval $\mathcal{I}$ of $\mathbb{R}$, we denote by $\overline{\mathcal{I}}$ (resp. $\stackrel{\mathcal{I}}{\text { ) }}$ its closure (resp. its interior). The scalar product $\langle\cdot, \cdot\rangle$ in $\mathscr{H}$ is right linear and $\|\cdot\|$ denotes the corresponding norm and also the norm in $\mathcal{B}(\mathscr{H})$, the space of bounded operators on $\mathscr{H}$. Let $A$ be a self-adjoint operator. Let $T$ be a closed operator. The form $[T, A]$ is defined on $(\mathcal{D}(A) \cap \mathcal{D}(T)) \times(\mathcal{D}(A) \cap \mathcal{D}(T))$ by

$$
\langle f,[T, A] g\rangle:=\left\langle T^{*} f, A g\right\rangle-\langle A f, T g\rangle .
$$


If $T$ is a bounded operator on $\mathscr{H}$ and $k \in \mathbb{N}$, we say that $T \in \mathcal{C}^{k}(A)$ if, for all $f \in \mathscr{H}$, the map $\mathbb{R} \ni t \mapsto e^{i t A} T e^{-i t A} f \in \mathscr{H}$ has the usual $\mathcal{C}^{k}$ regularity. The following characterization is available.

Proposition 2.1. ([ABG, p. 250]). Let $T \in \mathcal{B}(\mathscr{H})$. Are equivalent:

(1) $T \in \mathcal{C}^{1}(A)$.

(2) The form $[T, A]$ defined on $\mathcal{D}(A) \times \mathcal{D}(A)$ extends to a bounded form on $\mathscr{H} \times \mathscr{H}$ associated to a bounded operator denoted by $\operatorname{ad}_{A}^{1}(T):=[T, A]_{\mathrm{o}}$.

(3) $T$ preserves $\mathcal{D}(A)$ and the operator $T A-A T$, defined on $\mathcal{D}(A)$, extends to a bounded operator on $\mathscr{H}$.

It follows that $T \in \mathcal{C}^{k}(A)$ if and only if the iterated commutators $\operatorname{ad}_{A}^{p}(T):=$ $\left[\operatorname{ad}_{A}^{p-1}(T), A\right]_{\circ}$ are bounded for $p \leq k$. In particular, for $T \in \mathcal{C}^{1}(A), T \in \mathcal{C}^{2}(A)$ if and only if $[T, A]_{\circ} \in \mathcal{C}^{1}(A)$.

Let $H$ be a self-adjoint operator and $\mathcal{I}$ be an open interval. As in the Introduction (Section 1 ), we say that $H$ is locally of class $\mathcal{C}^{k}(A)$ on $\mathcal{I}$, we write $H \in \mathcal{C}_{\mathcal{I}}^{k}(A)$, if, for all $\varphi \in \mathcal{C}_{c}^{\infty}(\mathcal{I}), \varphi(H) \in \mathcal{C}^{k}(A)$.

It turns out that $T \in \mathcal{C}^{k}(A)$ if and only if, for a $z$ outside $\sigma(T)$, the spectrum of $T$, $(T-z)^{-1} \in \mathcal{C}^{k}(A)$. It is natural to say that $H \in \mathcal{C}^{k}(A)$ if $(H-z)^{-1} \in \mathcal{C}^{k}(A)$ for some $z \notin \sigma(H)$. In that case, $(H-z)^{-1} \in \mathcal{C}^{k}(A)$, for all $z \notin \mathbb{R}$. This regularity is stronger than the local one as asserted in the following

Proposition 2.2. ([ABG, p. 244]) If $H \in \mathcal{C}^{k}(A)$ then $H \in \mathcal{C}_{\mathcal{I}}^{k}(A)$ for all open interval $\mathcal{I}$ of $\mathbb{R}$.

Next we recall Proposition 2.1 in [GJ] which gives a sufficient condition to get the $\mathcal{C}^{1}(A)$ regularity for finite range operators.

Proposition 2.3. ([GJ]) If $f, g \in \mathcal{D}(A)$, then the rank one operator $|f\rangle\langle g|: h \mapsto$ $\langle g, h\rangle f$ is in $\mathcal{C}^{1}(A)$.

For $\rho \in \mathbb{R}$, let $\mathcal{S}^{\rho}$ be the class of functions $\varphi \in \mathcal{C}^{\infty}(\mathbb{R})$ such that

$$
\forall k \in \mathbb{N}, \quad C_{k}(\varphi):=\sup _{t \in \mathbb{R}}\langle t\rangle^{-\rho+k}\left|\varphi^{(k)}(t)\right|<\infty .
$$

Here $\varphi^{(k)}$ denotes the $k$ th derivative of $\varphi$. Equipped with the semi-norms defined by $(2.2), \mathcal{S}^{\rho}$ is a Fréchet space. We recall the following result from [DG] on almost analytic extension.

Proposition 2.4. ([DG]) Let $\varphi \in \mathcal{S}^{\rho}$ with $\rho \in \mathbb{R}$. There is a smooth function $\varphi^{\mathbb{C}}: \mathbb{C} \rightarrow \mathbb{C}$, called an almost analytic extension of $\varphi$, such that, for all $l \in \mathbb{N}$,

$$
\begin{aligned}
\left.\varphi^{\mathbb{C}}\right|_{\mathbb{R}}=\varphi, \quad & \left|\partial_{\bar{z}} \varphi^{\mathbb{C}}(z)\right| \leq c_{1}\langle\operatorname{Re}(z)\rangle^{\rho-1-l}|\operatorname{Im}(z)|^{l}, \\
& \operatorname{supp} \varphi^{\mathbb{C}} \subset\left\{x+i y ;|y| \leq c_{2}\langle x\rangle\right\}, \\
& \varphi^{\mathbb{C}}(x+i y)=0, \text { if } x \notin \operatorname{supp} \varphi,
\end{aligned}
$$

for constants $c_{1}, c_{2}$ depending on the semi-norms (2.2) of $\varphi$ in $\mathcal{S}^{\rho}$.

Next we recall Helffer-Sjöstrand's functional calculus (cf., [HeS, DG]). For $\rho<0$, $k \in \mathbb{N}$, and $\varphi \in \mathcal{S}^{\rho}$, the bounded operators $\varphi^{(k)}(A)$ can be recovered by

$$
\varphi^{(k)}(A)=\frac{i(k !)}{2 \pi} \int_{\mathbb{C}} \partial_{\bar{z}} \varphi^{\mathbb{C}}(z)(z-A)^{-1-k} d z \wedge d \bar{z},
$$


where the integral exists in the norm topology, by (2.3) with $l=1$. For $\rho \geq 0$, we rely on the following approximation:

Proposition 2.5. ([GJ]) Let $\rho \geq 0$ and $\varphi \in \mathcal{S}^{\rho}$. Let $\chi \in \mathcal{C}_{c}^{\infty}(\mathbb{R})$ with $\chi=1$ near 0 and $0 \leq \chi \leq 1$, and, for $R>0$, let $\chi_{R}(t)=\chi(t / R)$. For $f \in \mathcal{D}\left(\langle A\rangle^{\rho}\right)$, there exists

$$
\varphi^{(k)}(A) f=\lim _{R \rightarrow+\infty} \frac{i}{2 \pi} \int_{\mathbb{C}} \partial_{\bar{z}}\left(\varphi \chi_{R}\right)^{\mathbb{C}}(z)(z-A)^{-1-k} f d z \wedge d \bar{z} .
$$

The r.h.s. converges for the norm in $\mathscr{H}$. It is independent of the choise of $\chi$.

Notice that, for some $c>0$ and $s \in[0 ; 1]$, there exists some $C>0$ such that, for all $z=x+i y \in\{a+i b|0<| b \mid \leq c\langle a\rangle\}$ (like in (2.4)),

$$
\left\|\langle A\rangle^{s}(A-z)^{-1}\right\| \leq C\langle x\rangle^{s} \cdot|y|^{-1} .
$$

Observing that the self-adjointness assumption on $B$ is useless, we pick from [GJ, $\mathrm{Mø}]$ the following result in two parts.

Proposition 2.6. ([GJ, Mø]) Let $k \in \mathbb{N}^{*}, \rho<k, \varphi \in \mathcal{S}^{\rho}$, and $B$ be a bounded operator in $\mathcal{C}^{k}(A)$. As forms on $\mathcal{D}\left(\langle A\rangle^{k-1}\right) \times \mathcal{D}\left(\langle A\rangle^{k-1}\right)$,

$$
\begin{aligned}
{[\varphi(A), B]=} & \sum_{j=1}^{k-1} \frac{1}{j !} \varphi^{(j)}(A) \operatorname{ad}_{A}^{j}(B) \\
& +\frac{i}{2 \pi} \int_{\mathbb{C}} \partial_{\bar{z}} \varphi^{\mathbb{C}}(z)(z-A)^{-k} \operatorname{ad}_{A}^{k}(B)(z-A)^{-1} d z \wedge d \bar{z}
\end{aligned}
$$

In particular, if $\rho \leq 1$, then $B \in \mathcal{C}^{1}(\varphi(A))$.

The rest of the previous expansion is estimated in

Proposition 2.7. ([GJ, Mø]) Let $B \in \mathcal{C}^{k}(A)$ bounded. Let $\varphi \in \mathcal{S}^{\rho}$, with $\rho<k$. Let $I_{k}(\varphi)$ be the rest of the development of order $k(2.9)$ of $[\varphi(A), B]$, namely (2.10). Let $s, s^{\prime} \geq 0$ such that $s^{\prime}<1, s<k$, and $\rho+s+s^{\prime}<k$. Then, for $\varphi$ staying in a bounded subset of $\mathcal{S}^{\rho},\langle A\rangle^{s} I_{k}(\varphi)\langle A\rangle^{s^{\prime}}$ is bounded and there exists a $A$ and $\varphi$ independent constant $C>0$ such that $\left\|\langle A\rangle^{s} I_{k}(\varphi)\langle A\rangle^{s^{\prime}}\right\| \leq C\left\|\operatorname{ad}_{A}^{k}(B)\right\|$.

We refer to $[\mathrm{BG}]$ for some generalization of Propositions 2.6 and 2.7 to the case where $B$ is unbounded and $[A, B]$ 。 is bounded.

2.2. Pseudodifferential calculus. In this subsection, we briefly review some basic facts about pseudodifferential calculus that we need in the treatment of Schrödinger operators. We refer to [Hö3] [Chapters 18.1, 18.4, 18.5, and 18.6] for a traditional study of the subject but also to [Bo1, Bo2, BC, Le] for a modern and powerful version. Other results are presented in Appendix A and B.

Denote by $\mathcal{S}(M)$ the Schwartz space on the space $M$ and by $\mathcal{F}$ the Fourier transform on $\mathbb{R}^{d}$ given by

$$
\mathcal{F} u(\xi):=(2 \pi)^{-d} \int_{\mathbb{R}^{d}} e^{-i x \cdot \xi} u(x) d x,
$$


for $\xi \in \mathbb{R}^{d}$ and $u \in \mathcal{S}\left(\mathbb{R}^{d}\right)$. For test functions $u, v \in \mathcal{S}\left(\mathbb{R}^{d}\right)$, let $\Omega(u, v)$ and $\Omega^{\prime}(u, v)$ be the functions in $\mathcal{S}\left(\mathbb{R}^{2 d}\right)$ defined by

$$
\begin{aligned}
\Omega(u, v)(x, \xi) & :=\bar{v}(x) \mathcal{F} u(\xi) e^{i x \cdot \xi} \\
\Omega^{\prime}(u, v)(x, \xi) & :=(2 \pi)^{-d} \int_{\mathbb{R}^{d}} u(x-y / 2) \bar{v}(x+y / 2) e^{-i y \cdot \xi} d y,
\end{aligned}
$$

respectively. Given a distribution $b \in \mathcal{S}^{\prime}\left(T^{*} \mathbb{R}^{d}\right)$, the formal quantities

$$
\begin{array}{r}
(2 \pi)^{-d} \int_{\mathbb{R}^{3 d}} e^{i(x-y) \cdot \xi} b(x, \xi) v(x) u(y) d x d y d \xi \\
(2 \pi)^{-d} \int_{\mathbb{R}^{3 d}} e^{i(x-y) \cdot \xi} b((x+y) / 2, \xi) u(x) u(y) d x d y d \xi
\end{array}
$$

are defined by the duality brackets $\langle b, \Omega(u, v)\rangle$ and $\left\langle b, \Omega^{\prime}(u, v)\right\rangle$, respectively. They define continuous operators from $\mathcal{S}\left(\mathbb{R}^{d}\right)$ to $\mathcal{S}^{\prime}\left(\mathbb{R}^{d}\right)$ that we denote by $\operatorname{Op} b\left(x, D_{x}\right)$ and $b^{w}\left(x, D_{x}\right)$ respectively. Sometimes we simply write $\mathrm{Op} b$ and $b^{w}$, respectively. Choosing on the phase space $T^{*} \mathbb{R}^{d}$ a metric $g$ and a weight function $m$ with appropriate properties (cf., admissible metric and weight in [Le]), let $S(m, g)$ be the space of smooth functions on $T^{*} \mathbb{R}^{d}$ such that, for all $k \in \mathbb{N}$, there exists $c_{k}>0$ so that, for all $\mathrm{x}^{*}=(x, \xi) \in T^{*} \mathbb{R}^{d}$, all $\left(t_{1}, \cdots, t_{k}\right) \in\left(T^{*} \mathbb{R}^{d}\right)^{k}$,

$$
\left|a^{(k)}\left(\mathrm{x}^{*}\right) \cdot\left(t_{1}, \cdots, t_{k}\right)\right| \leq c_{k} m\left(x^{*}\right) g_{x^{*}}\left(t_{1}\right)^{1 / 2} \cdots g_{x^{*}}\left(t_{k}\right)^{1 / 2} .
$$

Here, $a^{(k)}$ denotes the $k$-th derivative of $a$. We equip the space $S(m, g)$ with the semi-norms $\|\cdot\|_{\ell, S(m, g)}$ defined by $\max _{0 \leq k \leq \ell} c_{k}$, where the $c_{k}$ are the best constants in (2.11). $S(m, g)$ is a Fréchet space. The space of operators $\operatorname{Op} b\left(x, D_{x}\right)$ (resp. $\left.b^{w}\left(x, D_{x}\right)\right)$ when $b \in S(m, g)$ has nice properties (cf., [Hö3, Le]). Defining $\mathrm{x}^{*}=(x, \xi) \in T^{*} \mathbb{R}^{d}$, we stick here to the following metrics

$$
g_{\mathrm{x}^{*}}:=\frac{d x^{2}}{\langle x\rangle^{2}}+\frac{d \xi^{2}}{\langle\xi\rangle^{2}} \quad \text { and } \quad\left(g_{0}\right)_{\mathrm{x}^{*}}:=d x^{2}+\frac{d \xi^{2}}{\langle\xi\rangle^{2}}
$$

and to weights of the form, for $p, q \in \mathbb{R}$,

$$
m\left(\mathrm{x}^{*}\right):=\langle x\rangle^{p}\langle\xi\rangle^{q} .
$$

The gain of the calculus associated to each metric in (2.12) is given respectively by

$$
h\left(\mathrm{x}^{*}\right):=\langle x\rangle^{-1}\langle\xi\rangle^{-1} \text { and } h_{0}\left(\mathrm{x}^{*}\right)=\langle\xi\rangle^{-1} \text {. }
$$

We note that $S(m, g) \subset S\left(m, g_{0}\right)$ with continuous injection. Take weights $m_{1}, m_{2}$ as in (2.13), let $\tilde{g}$ be $g$ or $g_{0}$, and denote by $\tilde{h}$ the gain of $\tilde{g}$. For any $a \in S\left(m_{1}, \tilde{g}\right)$ and $b \in S\left(m_{2}, \tilde{g}\right)$, there are a symbol $a \#{ }_{r} b \in S\left(m_{1} m_{2}, \tilde{g}\right)$ and a symbol $a \# b \in$ $S\left(m_{1} m_{2}, \tilde{g}\right)$ such that Op $a \mathrm{Op} b=\mathrm{Op}\left(a \#_{r} b\right)$ and $a^{w} b^{w}=(a \# b)^{w}$. The maps $(a, b) \mapsto a \#_{r} b$ and $(a, b) \mapsto a \# b$ are continuous and so are also $(a, b) \mapsto a \#_{r} b-a b \in$ $S\left(m_{1} m_{2} \tilde{h}, \tilde{g}\right)$ and $(a, b) \mapsto a \# b-a b \in S\left(m_{1} m_{2} \tilde{h}, \tilde{g}\right)$. If $a \in S\left(m_{1}, \tilde{g}\right)$, there exists $c \in S\left(m_{1}, \tilde{g}\right)$ such that $a^{w}=$ Op $c$. The maps $a \mapsto c$ and $a \mapsto c-a \in S\left(m_{1} m_{2} \tilde{h}, \tilde{g}\right)$ are continuous. If $a \in S(1, \tilde{g}), a^{w}$ and Op $a$ are bounded on $\mathrm{L}^{2}\left(\mathbb{R}^{d}\right)$. For $a \in S(1, \tilde{g})$,

$$
\text { Op } a \text { is compact } \Longleftrightarrow a^{w} \text { is compact } \Longleftrightarrow \lim _{\left|\mathrm{x}^{*}\right| \rightarrow \infty} a\left(\mathrm{x}^{*}\right)=0 \text {. }
$$




\section{Weighted Mourre theory.}

In this section, we present our new strategy to get the LAP (1.2). As in [GJ] (see also $[\mathrm{CGH}]$ ), we consider a more general version of the LAP, namely the LAP for the reduced resolvent (see (3.1) below). First we make use of a kind of weighted Weyl sequence introduced in [GJ], that we call "special sequence". Then we present an adapted version of the method introduced in [Gé] and based on energy estimates. Both methods are quite close, the latter having the advantage to give an idea of the size of the l.h.s. of (1.2) (resp. (3.1)).

3.1. Reduced resolvent. Let $P$ be the orthogonal projection onto the pure point spectral subspace of $H$ and $P^{\perp}=1-P$. For $s \geq 0$ and $\mathcal{I}^{\prime}$ an interval of $\mathbb{R}$, we say that the reduced LAP, respectively to the triplet $\left(\mathcal{I}^{\prime}, s, A\right)$, holds true for $H$ if

$$
\sup _{\operatorname{Re} z \in \mathcal{I}^{\prime}, \operatorname{Im} z \neq 0}\left\|\langle A\rangle^{-s}(H-z)^{-1} P^{\perp}\langle A\rangle^{-s}\right\|<\infty .
$$

Let $\mathcal{I}$ be an interval in $\mathbb{R}$ containing $\mathcal{I}^{\prime}$ in its interior. Since $(H-z)^{-1}\left(1-E_{\mathcal{I}}(H)\right)$ is uniformly bounded for $\operatorname{Re}(z) \in \mathcal{I}^{\prime}$ and $\operatorname{Im}(z) \neq 0,(3.1)$ is equivalent to the same estimate with $P^{\perp}$ replaced by $E_{\mathcal{I}}(H) P^{\perp}$. If no point spectrum is present in $\mathcal{I}$, then $(H-z)^{-1} E_{\mathcal{I}}(H) P^{\perp}=(H-z)^{-1}$ for $\operatorname{Re} z \in \mathcal{I}$ and (3.1) is equivalent to the usual LAP (1.2).

In $[\mathrm{CGH}]$ and more recently in [FMS2], it is shown that the reduced LAP can be derived from the Mourre estimate (1.1). In this case, it is well known that the point spectrum of $H$ is finite in $\mathcal{I}$ (but non empty in general, see [ABG, Mo]). In $[\mathrm{GJ}]$, the reduced LAP is deduced from a projected version of this Mourre estimate, namely

$$
P^{\perp} E_{\mathcal{I}}(H)[H, i A] E_{\mathcal{I}}(H) P^{\perp} \geq c E_{\mathcal{I}}(H) P^{\perp}+P^{\perp} K P^{\perp},
$$

for some compact operator $K$. In the proofs, one uses the compactness of $K$ and the fact that the strong limit

$$
s-\lim _{\delta \rightarrow 0} E_{] \lambda-\delta ; \lambda+\delta[}(H) P^{\perp}=0
$$

to derive from (3.2) a strict Mourre estimate (with $K=0$ ) on all small enough intervals inside $\mathcal{I}$. Notice that the traditional theory (cf., [ABG, Mo]) performs the same derivation. So both methods rely on some strict Mourre estimate. Here, to get the reduced LAP (3.1) as shown in Theorem 3.4 below, we also starts from a convenient strict estimate namely a strict, weighted, projected Mourre estimate like (1.4). We discuss the possibility to derive it from a more general one in Subsection 3.4. Since we work with projected estimates, we need some regularity of $P^{\perp}$ w.r.t. $A$.

3.2. Special sequences. We work in a larger framework.

Definition 3.1. Let $C$ be an injective, bounded, self-adjoint operator. Let $\mathcal{I}^{\prime}$ be an interval of $\mathbb{R}$.

(1) A special sequence $\left(f_{n}, z_{n}\right)_{n \in \mathbb{N}}$ for $H$ associated to $\left(\mathcal{I}^{\prime}, C\right)$ is a sequence $\left(f_{n}, z_{n}\right)_{n} \in(\mathcal{D}(H) \times \mathbb{C})^{\mathbb{N}}$ such that, for some $\eta \geq 0, \operatorname{Re}\left(z_{n}\right) \in \mathcal{I}^{\prime}, 0 \neq$ $\operatorname{Im}\left(z_{n}\right) \rightarrow 0,\left\|C f_{n}\right\| \rightarrow \eta, P^{\perp} f_{n}=f_{n},\left(H-z_{n}\right) f_{n} \in \mathcal{D}\left(C^{-1}\right)$, and $\| C^{-1}(H-$ $\left.z_{n}\right) f_{n} \| \rightarrow 0$. The limit $\eta$ is called the mass of the special sequence. 
(2) The reduced LAP, respectively to $\left(\mathcal{I}^{\prime}, C\right)$, holds true for $H$ if

$$
\sup _{\operatorname{Re} z \in \mathcal{I}^{\prime}, \operatorname{Im} z \neq 0}\left\|C(H-z)^{-1} P^{\perp} C\right\|<\infty .
$$

Notice that (3.4) for $C=\langle A\rangle^{-s}$ with $\left.s \in\right] 1 / 2 ; 1$ [ gives the LAP (3.1), thanks to Remark 1.2.

Proposition 3.2. Let $\mathcal{I}^{\prime}$ be an interval of $\mathbb{R}$. Take an injective, bounded, selfadjoint operator $C$ such that, for some $\chi$, a bounded, borelian function on $\mathbb{R}$ with $\chi=1$ near $\mathcal{I}^{\prime}$, the operator $C \chi(H) P^{\perp} C^{-1}$ extends to a bounded operator. Let $\theta$ be a borelian function on $\mathbb{R}$ such that $\theta \chi=\chi$. Then the reduced LAP (3.4) holds true if and only if, for all special sequence $\left(f_{n}, z_{n}\right)_{n}$ for $H$ associated to $\left(\mathcal{I}^{\prime}, C\right)$ such that $\theta(H) f_{n}=f_{n}$ for all $n$, the corresponding mass is zero.

Proof. Assume the LAP (3.4) true. Then, for any special sequence $\left(f_{n}, z_{n}\right)_{n}$ for $H$ associated to $\left(\mathcal{I}^{\prime}, C\right)$, for all $n$,

$$
\left\|C f_{n}\right\| \leq\left\|C\left(H-z_{n}\right)^{-1} P^{\perp} C\right\| \cdot\left\|C^{-1}\left(H-z_{n}\right) f_{n}\right\|,
$$

yielding $\eta=0$. Now assume the LAP (3.4) false. Then there exists some complex sequence $\left(z_{n}\right)$ such that $\operatorname{Re} z_{n} \in \mathcal{I}^{\prime}, \operatorname{Im} z_{n} \rightarrow 0$, and $\left\|C\left(H-z_{n}\right)^{-1} P^{\perp} C\right\| \rightarrow \infty$. Since $\left(\left(H-z_{n}\right)^{-1}(1-\chi)(H)\right)$ is uniformly bounded, we can find, for all $n$, nonzero $u_{n} \in \mathcal{H}$ and $0<\kappa_{n} \rightarrow 0$ such that

$$
\left\|C\left(H-z_{n}\right)^{-1} \chi(H) P^{\perp} C u_{n}\right\|=\left\|u_{n}\right\| / \kappa_{n} .
$$

We set $f_{n}:=\kappa_{n}\left(H-z_{n}\right)^{-1} \chi(H) P^{\perp} C u_{n} /\left\|u_{n}\right\|$. Notice that $\theta(H) f_{n}=P^{\perp} f_{n}=f_{n}$ and $\left\|C f_{n}\right\|=1$. Since $C \chi(H) P^{\perp} C^{-1}$ is bounded, $\chi(H) P^{\perp}$ preserves $\mathcal{D}\left(C^{-1}\right)$, the image of $C$. Thus $\left(H-z_{n}\right) f_{n} \in \mathcal{D}\left(C^{-1}\right)$, for all $n$. We conclude by noticing that $\left\|C^{-1}\left(H-z_{n}\right) f_{n}\right\| \leq \kappa_{n} \cdot\left\|C^{-1} \chi(H) P^{\perp} C\right\|=o(1)$.

Proposition 3.3. Let $\mathcal{I}^{\prime}, C$ be as in Proposition 3.2. Let $\left(f_{n}, z_{n}\right)_{n}$ be a special sequence for a self-adjoint operator $H$ associated to $\left(\mathcal{I}^{\prime}, C\right)$. For any bounded operator $B$, such that $C B C^{-1}$ extends to a bounded operator, $\lim _{n \rightarrow \infty}\left\langle f_{n},[H, B] f_{n}\right\rangle=0$.

Proof. Since $\left(f_{n}, z_{n}\right)_{n}$ is a special sequence and $C B C^{-1}$ is bounded, we obtain that $\left\langle\left(H-z_{n}\right) f_{n}, B f_{n}\right\rangle=o(1)$ and $\left\langle\left(H-z_{n}\right) f_{n}, f_{n}\right\rangle=o(1)$. Therefore, $2 i \operatorname{Im} z_{n}\left\|f_{n}\right\|^{2}=$ $\operatorname{Im}\left\langle\left(H-z_{n}\right) f_{n}, f_{n}\right\rangle=o(1)$. Hence

$$
\begin{aligned}
\left\langle f_{n},[H, i B] f_{n}\right\rangle & =\left\langle\left(H-\overline{z_{n}}\right) f_{n}, i B f_{n}\right\rangle-\left\langle B^{*} f_{n}, i\left(H-z_{n}\right) f_{n}\right\rangle \\
& =-2 i \operatorname{Im} z_{n} \cdot\left\langle f_{n}, i B f_{n}\right\rangle-2 \operatorname{Im}\left\langle\left(H-z_{n}\right) f_{n}, B f_{n}\right\rangle=o(1) .
\end{aligned}
$$

Theorem 3.4. Let $\mathcal{I}$ be an open interval and $\mathcal{I}^{\prime}$ be a closed sub-interval of $\mathcal{I}$. Let $B, C$ be two bounded self-adjoint operators, $C$ being injective. Assume that, for some bounded, borelian function $\chi$ on $\mathbb{R}$ with $\chi=1$ on $\mathcal{I}^{\prime}$ and $\operatorname{supp} \chi \subset \mathcal{I}$, $C \chi(H) P^{\perp} C^{-1}$ and $C B C^{-1}$ extend to bounded operators. Assume further that the following strict weighted projected Mourre estimate

$$
P^{\perp} E_{\mathcal{I}}(H)[H, i B] E_{\mathcal{I}}(H) P^{\perp} \geq P^{\perp} E_{\mathcal{I}}(H) C^{2} E_{\mathcal{I}}(H) P^{\perp}
$$

is satisfied. Then the LAP (3.4) on $\mathcal{I}^{\prime}$ holds true. 
Proof. Let $\left(f_{n}, z_{n}\right)_{n}$ be a special sequence for $H$ associated to $\left(\mathcal{I}^{\prime}, C\right)$ such that $E_{\mathcal{I}}(H) f_{n}=f_{n}$ for all $n$. By Proposition 3.2, it suffices to show that the mass $\eta$ of the special sequence is zero. Letting (3.5) act on both sides on $f_{n}$, we infer that $\left\langle f_{n},[H, i B] f_{n}\right\rangle \geq\left\|C f_{n}\right\|^{2}$. Now Proposition 3.3 yields $\eta=0$.

Proof of Theorem 1.1. Thanks to Remark 1.2, we may assume that $s \in] 1 / 2 ; 1[$ with $\left.\varepsilon:=2 s-1 \in] 0 ; \varepsilon_{0}\right]$. If, for $f \in \mathcal{D}(H)$ and for $E \in \mathcal{I}, H f=E f$ then, by $(1.4), 0 \geq$ $c_{1}\left\|\langle A\rangle^{-(1+\varepsilon) / 2} f\right\|^{2}$ and $f=0$. Thus $E_{\mathcal{I}}(H) P^{\perp}=E_{\mathcal{I}}(H)$ and (1.4) may be rewritten as (3.5) with $B=\varphi(A)$ and $C=\sqrt{c_{1}}\langle A\rangle^{-(1+\varepsilon) / 2}=\sqrt{c_{1}}\langle A\rangle^{-s}$. Notice that the function of $A$, given by $C B C^{-1}$, extends to a bounded operator. Let $\chi \in \mathcal{C}_{c}^{\infty}(\mathcal{I})$ such that $\chi=1$ on $\mathcal{I}^{\prime}$. Since $\chi(H) P^{\perp}=\chi(H) E_{\mathcal{I}}(H) P^{\perp}=\chi(H) E_{\mathcal{I}}(H)=\chi(H)$ and $H \in \mathcal{C}_{\mathcal{I}}^{1}(A), \chi(H) P^{\perp} \in \mathcal{C}^{1}(A)$. By Proposition 2.6, $\left[\chi(H) P^{\perp},\langle A\rangle^{s}\right]$ extends to a bounded operator. Thus, so does $\langle A\rangle^{-s} \chi(H) P^{\perp}\langle A\rangle^{s}=\langle A\rangle^{-s}\left[\chi(H) P^{\perp},\langle A\rangle^{s}\right]+$ $\chi(H) P^{\perp}$. This is also true for $C \chi(H) P^{\perp} C^{-1}$. By Theorem 3.4, (3.4) holds true. Since $E_{\mathcal{I}}(H) P^{\perp}=E_{\mathcal{I}}(H),(H-z)^{-1} P^{\perp}=(H-z)^{-1}$ for $\operatorname{Re} z \in \mathcal{I}^{\prime}$. Therefore (3.4) yields (1.2).

3.3. Energy estimates. Here we extend a little bit Gérard's method in [Gé]. We work in the general framework of Subsection 3.2 and get the following improvements of Theorem 3.4 and Theorem 1.1.

Theorem 3.5. Under the assumptions of Theorem 3.4, let $\sigma \in\{-1 ; 1\}$ and choose a real $\mu$ such that $\sigma B^{\prime} \geq 0$ with $B^{\prime}:=B+\mu$. Then

$$
\begin{aligned}
& \sup _{\operatorname{Re} z \in \mathcal{I}^{\prime},-\sigma \operatorname{Im} z>0}\left\|C(H-z)^{-1} P^{\perp} C\right\| \\
& \quad \leq 2 \cdot\left\|C B^{\prime} C^{-1}\right\| \cdot\left\|C^{-1} \chi(H) P^{\perp} C\right\|+d^{-1} \cdot\|1-\chi\|_{L^{\infty}} \cdot\|C\|^{2},
\end{aligned}
$$

where $d$ is the distance between the support of $1-\chi$ and $\mathcal{I}^{\prime}$.

Remark 3.6. Note that, for $\sigma$ and $B$ as in Theorem 3.5, one can always take $\mu=\sigma\|B\|$ to ensure $\sigma(B+\mu) \geq 0$.

Proof of Theorem 3.5. By functional calculus,

$$
\left\|C(H-z)^{-1}(1-\chi)(H) P^{\perp} C\right\| \leq d^{-1}\|1-\chi\|_{L^{\infty}} \cdot\|C\|^{2} .
$$

For $f \in \mathcal{H}$ and $z \in \mathbb{C}$ with $-\sigma \operatorname{Im} z>0$, let $u=(H-z)^{-1} \chi(H) P^{\perp} C f$. Notice that $E_{\mathcal{I}}(H) P^{\perp} u=u$. By (3.5) and a direct computation,

$$
\begin{aligned}
\|C u\|^{2} & \leq\left\langle u,\left[H, i B^{\prime}\right] u\right\rangle=2 \operatorname{Im}\left\langle B^{\prime} u,(H-z) u\right\rangle+2 \sigma \operatorname{Im} z\left\langle u, \sigma B^{\prime} u\right\rangle \\
& \leq 2 \operatorname{Im}\left\langle B^{\prime} u,(H-z) u\right\rangle,
\end{aligned}
$$

since $\sigma B^{\prime} \geq 0$. Recall that $C \chi(H) P^{\perp} C^{-1}$ is bounded. Thus $(H-z) u \in \mathcal{D}\left(C^{-1}\right)$. In particular, since $C B^{\prime} C^{-1}=C B C^{-1}+\mu$ is bounded,

$2^{-1}\|C u\|^{2} \leq \operatorname{Im}\left\langle C B^{\prime} C^{-1} C u, C^{-1}(H-z) u\right\rangle \leq\left\|C B^{\prime} C^{-1}\right\| \cdot\|C u\| \cdot\left\|C^{-1}(H-z) u\right\|$, yielding $\|C u\| \leq 2\left\|C B^{\prime} C^{-1}\right\| \cdot\left\|C^{-1} \chi(H) P^{\perp} C\right\| \cdot\|f\|$. Together with (3.7), this implies (3.6).

By combining the proof of Theorem 1.1 at the end of Subsection 3.2 with Theorem 3.5, we derive: 
Corollary 3.7. Under the assumptions of Theorem 1.1, take $s>1 / 2, \sigma \in\{-1 ; 1\}$, and $\chi \in \mathcal{C}_{c}^{\infty}(\mathcal{I})$ with $\chi=1$ on $\mathcal{I}^{\prime}$. Choose a real $\mu$ such that $\sigma(\varphi(A)+\mu) \geq 0$. Then the l.h.s. of (1.2) is bounded by the r.h.s. of (3.6) for $C=\sqrt{c_{1}}\langle A\rangle^{-s^{\prime}}$ and $B^{\prime}=\varphi(A)+\mu$ with $1 / 2<s^{\prime}<1,2 s^{\prime}-1 \leq \varepsilon_{0}$, and $s^{\prime} \leq s$.

3.4. Application. In practice, it is natural to try to derive a strict, weighted, projected Mourre estimate (1.4) from a similar estimate containing some compact perturbation. Precisely (1.4) should follow from

$$
\begin{aligned}
& P^{\perp} E_{\mathcal{I}}(H)[H, i \varphi(A)] E_{\mathcal{I}}(H) P^{\perp} \\
& \quad \geq E_{\mathcal{I}}(H) P^{\perp}\langle A\rangle^{-(1+\varepsilon) / 2}(c+K)\langle A\rangle^{-(1+\varepsilon) / 2} P^{\perp} E_{\mathcal{I}}(H),
\end{aligned}
$$

for some compact operator $K$ and $c>0$. But to remove the influence of $K$ using (3.3), we need to commute $P^{\perp} E_{\mathcal{I}}(H)$ (or a regularized version of it) through the weight $\langle A\rangle^{-(1+\varepsilon) / 2}$. We are able to do this in the following situation.

Corollary 3.8. Let $\mathcal{I}$ be an open interval. Assume that, for all $\theta \in \mathcal{C}_{c}^{\infty}(\mathcal{I} ; \mathbb{C})$, $P^{\perp} \theta(H) \in \mathcal{C}^{1}(A)$. Let $\left.\left.\varepsilon_{0} \in\right] 0 ; 1\right]$. Assume further that, for all $\left.\left.\varepsilon \in\right] 0 ; \varepsilon_{0}\right]$, there exist $c>0$ and a compact operator $K$ such that, for all $R \geq 1$, there exists a real bounded borelian function $\varphi_{R}$ such that the weighted projected Mourre estimate

$$
\begin{aligned}
& P^{\perp} E_{\mathcal{I}}(H)\left[H, i \varphi_{R}(A / R)\right] E_{\mathcal{I}}(H) P^{\perp} \\
& \quad \geq P^{\perp} E_{\mathcal{I}}(H)\langle A / R\rangle^{-(1+\varepsilon) / 2}(c+K)\langle A / R\rangle^{-(1+\varepsilon) / 2} E_{\mathcal{I}}(H) P^{\perp}
\end{aligned}
$$

is satisfied. Then, for any $s>1 / 2$ and for any compact sub-interval $\mathcal{I}^{\prime}$ of $\mathcal{I}$, the reduced $L A P(3.1)$ for $H$ respectively to $\left(\mathcal{I}^{\prime}, s, A\right)$ holds true.

Proof. By Remark 1.2, we may assume that $s \in] 1 / 2 ; 1[$ such that $\left.\varepsilon:=2 s-1 \in] 0 ; \varepsilon_{0}\right]$. By compactness of $\mathcal{I}^{\prime}$, it is sufficient to show that, for any $\lambda \in \mathcal{I}^{\prime},(3.1)$ holds true with $\mathcal{I}^{\prime}$ replaced by some open interval containing $\lambda$. It is enough to get (3.1) with $A$ replaced by $A / R$, for some $R \geq 1$. Let $\lambda \in \mathcal{I}^{\prime}$. Since $K$ in (3.9) is compact, we can use (3.3) to find $\chi \in \mathcal{C}_{c}^{\infty}(\mathcal{I} ; \mathbb{R})$ such that $\chi=1$ near $\lambda$ and $\left\|P^{\perp} \chi(H) K\right\| \leq c / 8$ (where $c$ appears in (3.9)). Let $\mathcal{I}_{1}$ be an open sub-interval of $\mathcal{I}^{\prime}$ containing $\lambda$. From (3.9), we get, for all $R \geq 1$,

$$
\begin{gathered}
P^{\perp} E_{\mathcal{I}_{1}}(H)\left[H, i \varphi_{R}(A / R)\right] E_{\mathcal{I}_{1}}(H) P^{\perp} \geq \\
P^{\perp} E_{\mathcal{I}_{1}}(H)\langle A / R\rangle^{-(1+\varepsilon) / 2} \cdot\left(3 c / 4+\left(1-P^{\perp} \chi(H)\right) K\left(1-P^{\perp} \chi(H)\right)\right) \\
\cdot\langle A / R\rangle^{-(1+\varepsilon) / 2} E_{\mathcal{I}_{1}}(H) P^{\perp} .
\end{gathered}
$$

Since $1-P^{\perp} \chi(H)=(1-\chi)(H)+P \chi(H)$,

$$
\begin{aligned}
P^{\perp} E_{\mathcal{I}_{1}}(H)\langle A / R\rangle^{-(1+\varepsilon) / 2}\left(1-P^{\perp} \chi(H)\right) & =-P^{\perp} E_{\mathcal{I}_{1}}(H)\left[\langle A / R\rangle^{-(1+\varepsilon) / 2}, P^{\perp} \chi(H)\right] \\
& =-P^{\perp} E_{\mathcal{I}_{1}}(H)\langle A / R\rangle^{-(1+\varepsilon) / 2} \cdot B_{R},
\end{aligned}
$$

where $\left\|B_{R}\right\|=O(1 / R)$ by Propositions 2.6 and 2.7 (with $k=1$ ). Taking $R$ large enough (but fixed), we derive from (3.10) the estimate

$$
P^{\perp} E_{\mathcal{I}_{1}}(H)\left[H, i \varphi_{R}(A / R)\right] E_{\mathcal{I}_{1}}(H) P^{\perp} \geq \frac{c}{2} P^{\perp} E_{\mathcal{I}_{1}}(H)\langle A / R\rangle^{-1-\varepsilon} E_{\mathcal{I}_{1}}(H) P^{\perp} .
$$

By Theorem 3.4, we obtain (3.4) with $C=\langle A / R\rangle^{-s}$ on some neighbourhood of $\lambda$, yielding (3.1) there with $A$ replaced by $A / R$. 


\section{Perturbed Wigner-Von Neumann potentials.}

In this section, we apply our new theory to some special Schrödinger operators (see Theorem 4.15). As explained in Section 1, we want to derive, on suitable intervals, a usual Mourre estimate (in Subsection 4.3) and a weighted, projected Mourre estimate (in Subsection 4.4) for the Schrödinger operator $H_{1}$, see (4.1).

4.1. Definitions and regularity. Let $d \in \mathbb{N}^{*}$. We denote by $\langle\cdot, \cdot\rangle$ and $\|\cdot\|$ the right linear scalar product and the norm in $\mathrm{L}^{2}\left(\mathbb{R}^{d}\right)$, the space of squared integrable, complex functions on $\mathbb{R}^{d}$. Recall that $H_{0}$ is the self-adjoint realization of the Laplace operator $-\Delta$ in $\mathrm{L}^{2}\left(\mathbb{R}^{d}\right)$ and that the Wigner-Von Neumann potential $W: \mathbb{R}^{d} \longrightarrow \mathbb{R}$ is defined by $W(x)=q(\sin k|x|) /|x|$, with $k>0$ and $q \in \mathbb{R}^{*}$. Now we add to $W$ the multiplication operator by the sum $V=V_{\mathrm{sr}}+V_{\mathrm{lr}}$ of real-valued functions, $V_{\mathrm{sr}}$ has short range, and $V_{\text {lr }}$ has long range. Precisely we require

Assumption 4.1. The functions $V_{\mathrm{lr}},\langle x\rangle V_{\mathrm{sr}}$, and the distribution $x \cdot \nabla V_{\mathrm{lr}}(x)$ belong to $\mathrm{L}^{\infty}\left(\mathbb{R}^{d}\right)$.

Under this assumption, on the Sobolev space $H^{2}\left(\mathbb{R}_{x}^{d}\right)$, the domain $\mathcal{D}\left(H_{0}\right)$ of $H_{0}$,

$$
H_{1}:=H_{0}+W+V=-\Delta+q|\cdot|^{-1} \sin (k|\cdot|)+V_{\mathrm{sr}}+V_{\mathrm{lr}}
$$

is self-adjoint. Let $P_{1}$ be the orthogonal projection onto its pure point spectral subspace and $P_{1}^{\perp}=1-P_{1}$.

Consider the strongly continuous one-parameter unitary group $\left\{\mathcal{W}_{t}\right\}_{t \in \mathbb{R}}$ acting by:

$$
\left(\mathcal{W}_{t} f\right)(x)=e^{d t / 2} f\left(e^{t} x\right) \text {, for all } f \in \mathrm{L}^{2}\left(\mathbb{R}_{x}^{d}\right) .
$$

This is the $C_{0}$-group of dilation. A direct computation shows that

$$
\mathcal{W}_{t} \mathrm{H}^{2}\left(\mathbb{R}_{x}^{d}\right) \subset \mathrm{H}^{2}\left(\mathbb{R}_{x}^{d}\right) \text {, for all } t \in \mathbb{R} .
$$

The generator of this group is the self-adjoint operator $A_{1}$, given by the closure of $\left(D_{x} \cdot x+x \cdot D_{x}\right) / 2$ on $\mathcal{C}_{c}^{\infty}\left(\mathbb{R}^{d}\right)$ in $\mathrm{L}^{2}\left(\mathbb{R}_{x}^{d}\right)$. For these reasons, the operator $A_{1}$ is called the generator of dilation.

The form $\left[W, i A_{1}\right]$ (defined on $\mathcal{D}\left(A_{1}\right) \times \mathcal{D}\left(A_{1}\right)$ ) extends to a bounded form associated to the multiplication operator by the function $W-W_{1}$, where

$$
W_{1}(x)=q k \cos (k|x|)=(q k / 2) \cdot\left(e^{i k|x|}+e^{-i k|x|}\right) .
$$

In particular, $W \in \mathcal{C}^{1}\left(A_{1}\right)$ by Proposition 2.1. Furthermore, we prove

Proposition 4.2. We have $H_{0} \in \mathcal{C}^{2}\left(A_{1}\right)$. Moreover, under Assumption 4.1, the form $\left[V_{\mathrm{s}}, i A_{1}\right]$, defined on $\mathcal{D}\left(A_{1}\right) \cap \mathcal{D}\left(H_{0}\right)$, extends to a bounded operator from $\mathrm{H}^{1}\left(\mathbb{R}_{x}^{d}\right)$ to $\mathrm{H}^{-1}\left(\mathbb{R}_{x}^{d}\right)$. In particular, $H_{1} \in \mathcal{C}^{1}\left(A_{1}\right)$.

Proof. We use Section 5. As form on $\mathcal{D}\left(A_{1}\right) \cap \mathcal{D}\left(H_{0}\right),\left[H_{0}, i A_{1}\right]=2 H_{0}$. In particular, (5.2) holds true with $A=A_{1}$ and $H=H_{0}$. By (4.3) and Theorem 5.2, $H_{0} \in \mathcal{C}^{1}\left(A_{1}\right)$ and $\left[H_{0}, i A_{1}\right]_{\circ}=2 H_{0}$. For $z \notin \mathbb{R}, R_{0}(z):=\left(H_{0}-z\right)^{-1}$ belongs to $\mathcal{C}^{1}\left(A_{1}\right)$. Using (5.3) with $A=A_{1}$ and $H=H_{0}$, we see that the form $\left[\left[R_{0}(z), i A_{1}\right]_{0}, i A_{1}\right]$ on $\mathcal{D}\left(A_{1}\right) \cap \mathcal{D}\left(H_{0}\right)$ extends to bounded one. Thus $R_{0}(z) \in \mathcal{C}^{2}\left(A_{1}\right)$ and $H_{0} \in \mathcal{C}^{2}\left(A_{1}\right)$. Since $\mathcal{D}\left(H_{1}\right)=\mathcal{D}\left(H_{0}\right)$ by Assumption 4.1, $H_{1} \in \mathcal{C}^{1}\left(A_{1}\right)$ follows from (4.3) and Theorem 5.2 if (5.2) holds true with $A=A_{1}$ and $H=H_{1}$. We consider the form 
$\left[H_{1}, i A_{1}\right]$ on $\mathcal{D}\left(A_{1}\right) \cap \mathcal{D}\left(H_{0}\right)$. It is the sum of $\left[H_{0}, i A_{1}\right]=2 H_{0}$, of the bounded terms $\left[W, i A_{1}\right]=W-W_{1}$ and $\left[V_{\mathrm{lr}}, i A_{1}\right]=x \cdot \nabla V_{\mathrm{lr}}$, and of

$\left\langle f,\left[V_{\mathrm{sr}}, i A_{1}\right] g\right\rangle=\left\langle V_{\mathrm{sr}} f, i A_{1} g\right\rangle-\left\langle A_{1} f, i V_{\mathrm{sr}} g\right\rangle$

$$
=\left\langle\langle x\rangle V_{\mathrm{sr}} f,\langle x\rangle^{-1}\left(x \cdot \nabla_{x}+d / 2\right) g\right\rangle+\left\langle\langle x\rangle^{-1}\left(x \cdot \nabla_{x}+d / 2\right) f,\langle x\rangle V_{\mathrm{sr}} g\right\rangle,
$$

for $f, g \in \mathcal{D}\left(A_{1}\right) \cap \mathcal{D}\left(H_{0}\right)$. Since $\langle x\rangle V_{\text {sr }}$ is bounded, (4.5) extends to a bounded operator from $\mathrm{H}^{1}\left(\mathbb{R}_{x}^{d}\right)$ to $\mathrm{H}^{-1}\left(\mathbb{R}_{x}^{d}\right)$ and also from $\mathrm{H}^{2}\left(\mathbb{R}_{x}^{d}\right)$ to $\mathrm{H}^{-2}\left(\mathbb{R}_{x}^{d}\right)$. This gives (5.2) for $(H, A)=\left(H_{1}, A_{1}\right)$. Thanks to (4.3) and to Theorem 5.2, $H_{1} \in \mathcal{C}^{1}\left(A_{1}\right)$.

4.2. Energy localization of oscillations. To prepare the derivation of Mourre estimates, we take advantage of some "smallness" of energy localizations of $W_{1}$ of the form $\theta\left(H_{0}\right) W_{1} \theta\left(H_{0}\right)$, extending a result by $[\mathrm{FH}]$ in dimension one. As seen in $[\mathrm{FH}]$, this term is not expected to be small if $\theta$ is localized near $k^{2} / 4$. Using pseudodifferential calculus, one have the same impression if $d \geq 2$ and if $\theta$ is supported in ]$k^{2} / 4 ;+\infty[$ (see Remark 4.5). However, if $\theta$ lives in a small enough compact interval $\mathcal{I} \subset] 0 ; k^{2} / 4[$, then the same smallness as in $[\mathrm{FH}]$ is valid as stated in Lemma 4.3 below. The proof combines an idea in $[\mathrm{FH}]$ with pseudodifferential calculus (see Subsection 2.2 for notation). In the sequel, we shall write $\hat{x}$ for $x /|x|$.

Lemma 4.3. Let $\lambda \in] 0 ; k^{2} / 4\left[\right.$. Recall that $g$ is given by (2.12) and $W_{1}$ by (4.4). Take $\chi_{1} \in \mathcal{C}^{\infty}\left(\mathbb{R}^{d}\right)$ such that $\chi_{1}=0$ near 0 and $\chi_{1}=1$ near infinity, and set $e_{ \pm}(x)=\chi_{1}(x) e^{ \pm i k|x|}$. For $\theta \in \mathcal{C}_{c}^{\infty}(\mathbb{R})$ with small enough support about $\lambda$, there exist symbols $b_{0}, b_{j, \sigma} \in \mathcal{S}\left(\langle x\rangle^{-1}\langle\xi\rangle^{-1}, g\right)$, for $j \in\{1 ; 2\}$ and $\sigma \in\{+,-\}$, such that

$$
\theta\left(H_{0}\right) W_{1} \theta\left(H_{0}\right)=b_{1,+}^{w} e_{+}+b_{1,-}^{w} e_{-}+\theta\left(H_{0}\right)\left(e_{+} b_{2,+}^{w}+e_{-} b_{2,-}^{w}\right)+b_{0}^{w} .
$$

In particular, $\left\langle A_{1}\right\rangle^{\varepsilon} \theta\left(H_{0}\right) W_{1} \theta\left(H_{0}\right)$ is compact on $\mathrm{L}^{2}\left(\mathbb{R}_{x}^{d}\right)$, for $\varepsilon \in[0 ; 1[$.

Remark 4.4. In dimension $d=1$, this result is proved in $[\mathrm{FH}]$ and it also holds true if $\lambda>k^{2} / 4$. Our proof below covers also this case.

Proof of Lemma 4.3. By pseudodifferential calculus, $\theta\left(H_{0}\right)\left(1-\chi_{1}\right) W_{1} \theta\left(H_{0}\right)=b_{0}^{w}$ with $b_{0} \in \mathcal{S}\left(\langle x\rangle^{-1}\langle\xi\rangle^{-1}, g\right)$. By (4.4) and the proof of Proposition A.1, we can find $\chi_{3} \in \mathcal{C}^{\infty}\left(\mathbb{R}^{d}\right)$ such that $\chi_{3}=0$ near 0 and $\chi_{3}=1$ near infinity, and $b_{j, \sigma} \in$ $\mathcal{S}\left(\langle x\rangle^{-1}\langle\xi\rangle^{-1}, g\right)$, for $j \in\{0 ; 2\}$ and $\sigma \in\{+,-\}$, such that

$$
\begin{aligned}
2(q k)^{-1} & \theta\left(H_{0}\right) \chi_{1} W_{1} \theta\left(H_{0}\right) \\
= & \theta\left(H_{0}\right)\left(\left(\theta\left(|\xi-k \hat{x}|^{2}\right) \chi_{3}(x)\right)^{w} e_{+}+\left(\theta\left(|\xi+k \hat{x}|^{2}\right) \chi_{3}(x)\right)^{w} e_{-}\right) \\
& +\theta\left(H_{0}\right)\left(b_{0,+}^{w} e_{+}+b_{0,-}^{w} e_{-}+e_{+} b_{2,+}^{w}+e_{-} b_{2,-}^{w}\right) \\
=\left(\theta\left(|\xi|^{2}\right) \theta(\mid \xi-\right. & \left.\left.\left.k \hat{x}\right|^{2}\right) \chi_{3}(x)\right)^{w} e_{+}+\left(\theta\left(|\xi|^{2}\right) \theta\left(|\xi+k \hat{x}|^{2}\right) \chi_{3}(x)\right)^{w} e_{-} \\
& +b_{1,+}^{w} e_{+}+b_{1,-}^{w} e_{-}+\theta\left(H_{0}\right)\left(e_{+} b_{2,+}^{w}+e_{-} b_{2,-}^{w}\right),
\end{aligned}
$$

by composition. Now we choose the support of $\theta$ small enough about $\lambda$ such that $\theta\left(|\xi|^{2}\right) \theta\left(|\xi-k \hat{x}|^{2}\right)=0=\theta\left(|\xi|^{2}\right) \theta\left(|\xi+k \hat{x}|^{2}\right)$, for all $x \neq 0$ and $\xi \in \mathbb{R}^{d}$. This is possible since $0 \leq \lambda<k^{2} / 4$, see Figure 1. Now (4.7) reduces to (4.6). By Appendix C, $\left\langle A_{1}\right\rangle^{\varepsilon}\left\langle D_{x}\right\rangle^{-\varepsilon}\langle x\rangle^{-\varepsilon}$ extends to a bounded operator. For $b \in \mathcal{S}\left(\langle x\rangle^{-1}\langle\xi\rangle^{-1}, g\right)$, there exists $b \in \mathcal{S}\left(\langle x\rangle^{\varepsilon-1}\langle\xi\rangle^{\varepsilon-1}, g\right)$ such that $\langle x\rangle^{\varepsilon}\left\langle D_{x}\right\rangle^{\varepsilon} b^{w}=b_{1}^{w}$ and $b_{1}^{w}$ is compact by (2.15). Using (4.6), this implies that $\left\langle A_{1}\right\rangle^{\varepsilon} \theta\left(H_{0}\right) W_{1} \theta\left(H_{0}\right)$ is compact since we can write $\theta\left(H_{0}\right) e_{+} b_{2,+}^{w}=\theta\left(H_{0}\right)\langle x\rangle^{-1} e_{+}\langle x\rangle b_{2,+}^{w}$ with $\langle x\rangle b_{2,+}^{w}$ bounded and $\theta\left(H_{0}\right)\langle x\rangle^{-1}=$ $b^{w}$ with $b \in \mathcal{S}\left(\langle x\rangle^{-1}\langle\xi\rangle^{-1}, g\right)$. 


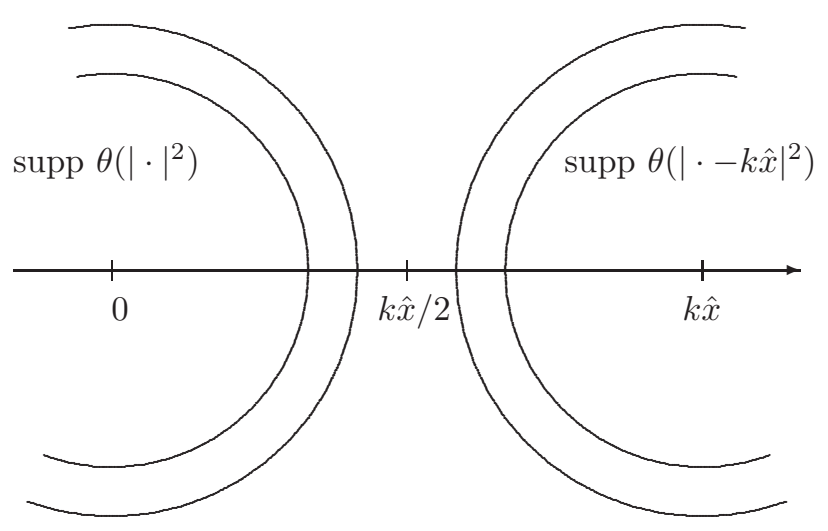

FiguRE 1. $\operatorname{supp} \theta \subset] 0, k^{2} / 4[$.

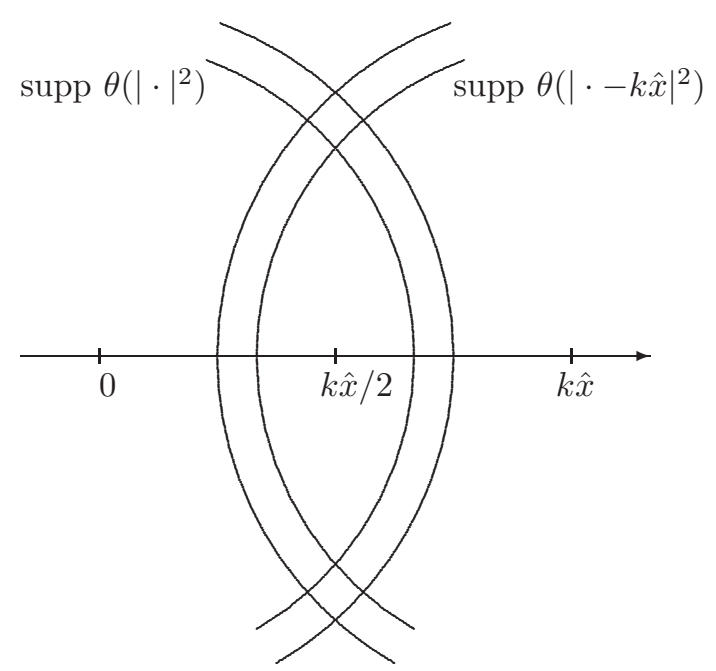

FiguRe 2. $\operatorname{supp} \theta \subset] k^{2} / 4,+\infty[$.

Remark 4.5. If $\lambda>k^{2} / 4$ and $d>1$, the first two terms on the r.h.s. of (4.7) do not vanish anymore, see Figure 2. In this case, our proofs of the Mourre estimate (cf., Proposition 4.8) and of the strict, weighted Mourre estimate (cf., Subsection 4.4) do not work.

In dimension $d=1$, we note that the first two terms on the r.h.s. of (4.7) do vanish as soon as $\lambda \neq k^{2} / 4$. See Figures 1 and 2 and recall that $\xi$ is co-linear to $\hat{x}$. We recover a result in $[\mathrm{FH}]$.

4.3. Usual Mourre estimate. Now we derive the Mourre estimate (1.1) below $k^{2} / 4$ under the following strengthening of Assumption 4.1: 
Assumption 4.6. The functions $V_{\mathrm{lr}},\langle x\rangle V_{\mathrm{sr}}$, and the distribution $x \cdot \nabla V_{\mathrm{lr}}(x)$ belong to $\mathrm{L}^{\infty}\left(\mathbb{R}_{x}^{d}\right)$ and, as operator of multiplication, compact from $\mathrm{H}^{2}\left(\mathbb{R}_{x}^{d}\right)$ to $\mathrm{L}^{2}\left(\mathbb{R}_{x}^{d}\right)$.

Lemma 4.7. Under Assumption 4.6, $\varphi\left(H_{1}\right)-\varphi\left(H_{0}\right)$ is compact from $\mathrm{H}^{2}\left(\mathbb{R}_{x}^{d}\right)$ to $\mathrm{L}^{2}\left(\mathbb{R}_{x}^{d}\right)$, for $\varphi \in \mathcal{C}_{c}^{\infty}(\mathbb{R})$.

Proof. Using (2.6), one has $\left(\varphi\left(H_{1}\right)-\varphi\left(H_{0}\right)\right)\left\langle H_{0}\right\rangle=$

$$
\frac{i}{2 \pi} \int_{\mathbb{C}} \partial_{\bar{z}} \varphi^{\mathbb{C}}(z)\left(z-H_{1}\right)^{-1}\left(W+V_{\mathrm{sr}}+V_{\mathrm{lr}}\right)\left(z-H_{0}\right)^{-1}\left\langle H_{0}\right\rangle d z \wedge d \bar{z} .
$$

For $z \notin \mathbb{R}$, the integrand is compact. Using (2.8), the integral converges in norm. Hence it is also compact.

Proposition 4.8. Under Assumption 4.6, for any open interval $\mathcal{I}$ with $\overline{\mathcal{I}} \subset$ ] $0 ; k^{2} / 4\left[\right.$, the Mourre estimate (1.1) holds true for $(H, A)=\left(H_{1}, A_{1}\right)$. In particular, the point spectrum $\sigma_{p p}\left(H_{1}\right)$ of $H_{1}$ is finite in $\mathcal{I}$.

Proof. It suffices to show (1.1) on some compact neighborhood of any $\lambda \in \mathcal{I}$. Take such a $\lambda \in \mathcal{I}$ and let $\theta \in \mathcal{C}_{c}^{\infty}(\mathcal{I} ;[0,1])$ such that $\theta=1$ near $\lambda$. Like in the proof of Proposition 4.2, as form on $\mathcal{D}\left(H_{0}\right) \cap \mathcal{D}\left(A_{1}\right) \times \mathcal{D}\left(H_{0}\right) \cap \mathcal{D}\left(A_{1}\right)$,

$$
\left[H_{0}+V, i A_{1}\right]=2 H_{0}-x \cdot \nabla V_{\mathrm{lr}}-\nabla \cdot x\langle x\rangle^{-1}\langle x\rangle V_{\mathrm{sr}}-\langle x\rangle V_{\mathrm{sr}}\langle x\rangle^{-1} x \cdot \nabla .
$$

We recall that $\left[W, i A_{1}\right]_{\circ}=W-W_{1}$. Hence $\left[H_{1}, i A_{1}\right]_{\circ}$ is bounded from $\mathcal{D}\left(H_{0}\right)=$ $\mathrm{H}^{2}\left(\mathbb{R}_{x}^{d}\right)$ to $\mathcal{D}\left(H_{0}\right)^{*}=\mathrm{H}^{-2}\left(\mathbb{R}_{x}^{d}\right)$. Moreover, by Lemma 4.7 , the bounded operator $\theta\left(H_{1}\right)\left[H_{1}, i A_{1}\right]_{\circ} \theta\left(H_{1}\right)$ is equal to $\theta\left(H_{0}\right)\left(2 H_{0}-W_{1}\right) \theta\left(H_{0}\right)$, up to some compact operator. By Lemma 4.3, we can choose the support of $\theta$ such that $\theta\left(H_{0}\right) W_{1} \theta\left(H_{0}\right)$ is compact. Thus, there exist $c>0$ and compact operators $K, K^{\prime}$ such that

$$
\theta\left(H_{1}\right)\left[H_{1}, A_{1}\right]_{\circ} \theta\left(H_{1}\right) \geq c \theta\left(H_{0}\right)^{2}+K^{\prime} \geq c \theta\left(H_{1}\right)^{2}+K .
$$

This yields the Mourre estimate (1.1) near $\lambda$.

As explained in Subsection 3.1, we need some information on possible eigenvalues embedded in the interval on which the LAP takes place. Recall that $P_{1}$ denotes the orthogonal projection onto the pure point spectral subspace of $H_{1}$.

Proposition 4.9. Under Assumption 4.6, take an open interval $\mathcal{I}$ with $\overline{\mathcal{I}} \subset] 0 ; k^{2} / 4[$ such that, for all $\mu \in \mathcal{I}$, $\operatorname{Ker}\left(H_{1}-\mu\right) \subset \mathcal{D}\left(A_{1}\right)$. Then $E_{\mathcal{I}}\left(H_{1}\right) P_{1} \in \mathcal{C}^{1}\left(A_{1}\right)$.

Proof. By Proposition 4.8, the point spectrum is finite in $\mathcal{I}$. Thus $E_{\mathcal{I}}\left(H_{1}\right) P_{1} \in$ $\mathcal{C}^{1}\left(A_{1}\right)$, by Proposition 2.3.

We now explain how to check the hypothesis $\operatorname{Ker}\left(H_{1}-\mu\right) \subset \mathcal{D}\left(A_{1}\right)$. The abstract Theorems given in [Ca, FMS1] do not apply here because of the low regularity of $H_{1}$ w.r.t. $A_{1}$, see the inclusions (5.6), the implication (5.7), and Proposition 5.4. For $j \in\{1 ; \cdots ; d\}$, the multiplication operator by $x_{j}$ in $\mathrm{L}^{2}\left(\mathbb{R}_{x}^{d}\right)$ is also denoted by $x_{j}$. As preparation, we show, using a Lithner-Agmon type equality, the following

Lemma 4.10. Let $n \in \mathbb{N}$. If $v \in \mathcal{C}^{2}\left(\mathbb{R}_{x}^{d}\right) \cap \mathrm{H}^{2}\left(\mathbb{R}_{x}^{d}\right) \cap \mathcal{D}\left(\langle x\rangle^{2 n}\right)$ then $\nabla_{x} v \in \mathcal{D}\left(\langle x\rangle^{n}\right)$.

Proof. Define $\Phi(x)=n \ln \langle x\rangle$ for $x \in \mathbb{R}^{d}$ and let $R>1$. Using Green's formula, we can show that

$$
\int_{|x| \leq R}\left|\nabla\left(e^{\Phi} v\right)\right|^{2} d x=a(R)+\operatorname{Re} \int_{|x| \leq R} e^{2 \Phi} \bar{v}\left(-\Delta v+|\nabla \Phi|^{2} v\right) d x
$$


where the term $a(R)$ contains surface integrals on $\{|x|=R\}$ and tends to 0 as $R \rightarrow \infty$, thanks to $v \in \mathcal{D}\left(\langle x\rangle^{2 n}\right)$ and $v \in \mathcal{C}^{2}\left(\mathbb{R}^{d}\right)$. Since $v \in \mathrm{H}^{2}\left(\mathbb{R}_{x}^{d}\right)$, the last term in (4.8) converges as $R \rightarrow \infty$, yielding $\nabla\left(e^{\Phi} v\right) \in \mathrm{L}^{2}\left(\mathbb{R}_{x}^{d}\right)$. Since $e^{\Phi} v \nabla \Phi \in \mathrm{L}^{2}\left(\mathbb{R}_{x}^{d}\right)$, $\langle x\rangle^{n} \nabla v=e^{\Phi} \nabla v \in \mathrm{L}^{2}\left(\mathbb{R}_{x}^{d}\right)$.

Lemma 4.11. Under Assumption 4.6, let $u \in \mathcal{C}^{2}\left(\mathbb{R}^{d}\right) \cap \mathrm{H}^{2}\left(\mathbb{R}_{x}^{d}\right)$ and $\left.\lambda \in\right] 0 ; k^{2} / 4[$ such that $\left(H_{1}-\lambda\right) u=0$. Then $u \in \mathcal{D}\left(A_{1}\right)$. Moreover, if $V_{\mathrm{lr}}=0$, then $u=0$.

Proof. By Proposition 4.8, the usual Mourre estimate holds true near $\lambda$. Thus, one can apply Theorem 2.1 in $[\mathrm{FH}]$. Therefore $u \in \mathcal{D}\left(\langle x\rangle^{n}\right)$, for all $n \in \mathbb{N}$. By Lemma 4.10, $D^{\alpha} u \in \mathcal{D}\left(\langle x\rangle^{n}\right)$, for all $n$ and all $\alpha \in \mathbb{N}^{d}$ with $|\alpha|=1$. In particular, $x \cdot \nabla u \in \mathrm{L}^{2}\left(\mathbb{R}_{x}^{d}\right)$ and $u \in \mathcal{D}\left(A_{1}\right)$. If $V_{\text {lr }}=0$, we can apply Theorem 14.7.2 in [Hö2] to $u$ yielding $u=0$.

Remark 4.12. If the potential $V=V_{\text {sr }}+V_{\text {lr }}$ belongs to $\mathcal{C}^{m}\left(\mathbb{R}^{d}\right)$ for some integer $m>d / 2$ then, by elliptic regularity, any eigenvector $u$ of $H_{1}$ belongs to $\mathcal{C}^{2}\left(\mathbb{R}^{d}\right)$. In particular, by Lemma 4.11, Proposition 4.9 applies to any open interval $\mathcal{I}$ such that $\overline{\mathcal{I}} \subset] 0 ; k^{2} / 4[$.

4.4. Weighted Mourre estimate. Here we establish for $H_{1}$ a projected, weighted Mourre estimate like (3.9) in order to prove a limiting absorption principle (cf., Theorem 4.15). To this end, we use the following assumption, which is stronger than Assumption 4.6.

Assumption 4.13. For some $\left.\left.\rho_{0} \in\right] 0,1\right]$, the functions $\langle x\rangle^{\rho_{0}} V_{\mathrm{lr}},\langle x\rangle^{1+\rho_{0}} V_{\mathrm{sr}}$, and the distribution $\langle x\rangle^{\rho_{0}} x \cdot \nabla V_{\mathrm{lr}}(x)$ belong to $\mathrm{L}^{\infty}\left(\mathbb{R}_{x}^{d}\right)$.

We start by strengthening Lemma 4.7.

Lemma 4.14. Under Assumption 4.13, for $\varepsilon \in\left[0 ; \rho_{0}\left[\right.\right.$ and $\varphi \in \mathcal{C}_{c}^{\infty}(\mathbb{R})$,

$$
\left(\varphi\left(H_{1}\right)-\varphi\left(H_{0}\right)\right)\left\langle A_{1}\right\rangle^{\varepsilon} \text { is compact from } \mathrm{L}^{2}\left(\mathbb{R}_{x}^{d}\right) \text { to } \mathrm{H}^{2}\left(\mathbb{R}_{x}^{d}\right) \text {. }
$$

Proof. For $z \notin \mathbb{R},\left(z-H_{0}\right)^{-1}=r_{z}^{w}$ where $r_{z}$ satisfies (B.3) with $m=\langle\xi\rangle^{2}$. By composition, we can find, for all $\ell \in \mathbb{N}, C_{\ell}>0$ and $N_{\ell} \in \mathbb{N}$ such that, for all $z \notin \mathbb{R}$,

$$
\left\|\langle x\rangle^{-\rho_{0}} \# r_{z} \#\left(\langle x\rangle^{\varepsilon}\langle\xi\rangle^{\varepsilon}\right)\right\|_{\ell, \mathcal{S}\left(\langle x\rangle^{\varepsilon-\rho_{0}}\langle\xi\rangle^{\varepsilon-2}, g\right)} \leq C_{\ell}\langle z\rangle^{N_{\ell}+1}|\operatorname{Im}(z)|^{-N_{\ell}-1} .
$$

Now thanks to Assumption 4.13, (2.15), (2.3), (2.4), and (2.5), we infer that

$$
\begin{aligned}
\left\langle H_{1}\right\rangle\left(\varphi\left(H_{1}\right)-\varphi\left(H_{0}\right)\right)\langle x\rangle^{\varepsilon}\left\langle D_{x}\right\rangle^{\varepsilon}=\frac{i}{2 \pi} \int_{\mathbb{C}} \partial_{\bar{z}} \varphi^{\mathbb{C}}(z)\left\langle H_{1}\right\rangle\left(z-H_{1}\right)^{-1} \\
\left(W+V_{\mathrm{sr}}+V_{\mathrm{lr}}\right)\langle x\rangle^{\rho_{0}}\langle x\rangle^{-\rho_{0}}\left(z-H_{0}\right)^{-1}\langle x\rangle^{\varepsilon}\left\langle D_{x}\right\rangle^{\varepsilon} d z \wedge d \bar{z}
\end{aligned}
$$

is a compact operator, as norm convergent integral of compact operators. To conclude, we recall $\langle x\rangle^{-\varepsilon}\left\langle D_{x}\right\rangle^{-\varepsilon}\left\langle A_{1}\right\rangle^{\varepsilon}$ is bounded by Lemma C.1.

The main result on Schrödinger operators with oscillating potential is

Theorem 4.15. Let $\lambda \in] 0 ; k^{2} / 4[$ and suppose that Assumption 4.13 is satisfied. Take a small enough, open interval $\mathcal{I} \subset] 0 ; k^{2} / 4[$ about $\lambda$ such that, for all $\mu \in \mathcal{I}$, $\operatorname{Ker}\left(H_{1}-\mu\right) \subset \mathcal{D}\left(A_{1}\right)$. Then, for any $s>1 / 2$ and any interval $\mathcal{I}^{\prime} \subset \overline{\mathcal{I}^{\prime}} \subset \mathcal{I}$, the reduced $L A P(3.1)$ for $H_{1}$ respectively to $\left(\mathcal{I}^{\prime}, s, A_{1}\right)$ holds true. 
Remark 4.16. Of course, a compactness argument shows that we can remove the smallness condition on $\mathcal{I}$. We also get an estimate like in (3.6) in Theorem 3.5. If $d=1$, the proof of Theorem 4.15 works also if $\lambda>k^{2} / 4$, by Remark 4.4 .

Under Assumption 4.13, Theorem 4.15 ensures that $H_{1}$ has no singular spectrum above $\mathcal{I}$. In dimension $d=1$, for $V_{\mathrm{lr}}=0$ but under a weaker assumption on $V_{\mathrm{sr}}$, this result was already obtained in $[\mathrm{Ki}, \mathrm{Re}]$ (see references therein). Our long-range result seems to be new, even in dimension 1.

If $\mathcal{I}$ contains an eigenvalue $\mu$ of $H_{1}$, the condition $\operatorname{Ker}\left(H_{1}-\mu\right) \subset \mathcal{D}\left(A_{1}\right)$ is satisfied if $V=V_{\mathrm{sr}}+V_{\mathrm{lr}}$ is smooth enough (cf., Proposition 4.9 and Remark 4.12).

If one sets $q=0$, i.e. if one removes the potential $W, H_{1}$ has no embedded eigenvalue (cf., $[\mathrm{FH}]$ ). The following proof of Theorem 4.15 works for each compact interval $\mathcal{I}^{\prime} \subset(0, \infty)$ and gives the $\operatorname{LAP}(1.2)$ with $(H, A)=\left(H_{1}, A_{1}\right)$. This is a well-known result that can be obtained by the involved versions of the Mourre theory which are exposed in $[\mathrm{ABG}, \mathrm{Sa}]$. But the technics of [Mo, Gé] do not apply, since the needed regularity condition $H_{1} \in \mathcal{C}^{2}\left(A_{1}\right)$ is not always satisfied under Assumption 4.13.

Proof of Theorem 4.15: Let $\theta, \chi, \tau \in \mathcal{C}_{c}^{\infty}(] 0 ; k^{2} / 4[)$ such that $\tau \chi=\chi, \chi \theta=\theta$, and $\theta=1$ near $\mathcal{I}$. Later we shall adjust the size of the support of $\chi$. By Proposition 4.2 and $(5.7), \chi\left(H_{1}\right) \in \mathcal{C}^{1}\left(A_{1}\right)$. Since $E_{\mathcal{I}}\left(H_{1}\right) P_{1} \in \mathcal{C}^{1}\left(A_{1}\right)$ by Proposition 4.9, $\chi\left(H_{1}\right) P_{1}^{\perp}=\chi\left(H_{1}\right)-\chi\left(H_{1}\right) E_{\mathcal{I}}\left(H_{1}\right) P_{1}$ belongs to $\mathcal{C}^{1}\left(A_{1}\right)$.

Let $s \in] 1 / 2 ; 1[$. As in [Gé], we define $\psi: \mathbb{R} \longrightarrow \mathbb{R}$ by

$$
\psi(t):=\int_{-\infty}^{t}\langle u\rangle^{-2 s} d u .
$$

Note that $\psi \in \mathcal{S}^{0}$ and is in particular bounded. Let $R \geq 1$. As forms, using the fact that $H_{1} \tau\left(H_{1}\right)$ is a bounded operator and belongs to $\mathcal{C}^{1}\left(A_{1}\right)$ and using $(2.9)$,

$$
\begin{gathered}
F:=P_{1}^{\perp} \theta\left(H_{1}\right)\left[H_{1}, i \psi\left(A_{1} / R\right)\right] \theta\left(H_{1}\right) P_{1}^{\perp}=P_{1}^{\perp} \theta\left(H_{1}\right)\left[H_{1} \tau\left(H_{1}\right), i \psi\left(A_{1} / R\right)\right] \theta\left(H_{1}\right) P_{1}^{\perp} \\
=\frac{i}{2 \pi} \int_{\mathbb{C}} \partial_{\bar{z}} \psi^{\mathbb{C}}(z) P_{1}^{\perp} \theta\left(H_{1}\right)\left(z-A_{1} / R\right)^{-1}\left[H_{1} \tau\left(H_{1}\right), i A_{1} / R\right]_{\circ} \\
\left(z-A_{1} / R\right)^{-1} \theta\left(H_{1}\right) P_{1}^{\perp} d z \wedge d \bar{z} .
\end{gathered}
$$

Next to $P_{1}^{\perp} \theta\left(H_{1}\right)$ we let appear $\chi\left(H_{1}\right) P_{1}^{\perp}$ and commute it with $\left(z-A_{1} / R\right)^{-1}$. Since $\chi\left(H_{1}\right) P_{1}^{\perp} \in \mathcal{C}^{1}\left(A_{1}\right)$, we obtain, using (2.3), (2.4), and (2.8), for some uniformly bounded operator $B_{1}$ w.r.t. $R \geq 1$,

$$
\begin{gathered}
F=\frac{i}{2 \pi} \int_{\mathbb{C}} \partial_{\bar{z}} \psi^{\mathbb{C}}(z) P_{1}^{\perp} \theta\left(H_{1}\right)\left(z-A_{1} / R\right)^{-1} P_{1}^{\perp} \chi\left(H_{1}\right)\left[H_{1} \tau\left(H_{1}\right), i A_{1} / R\right]_{\circ} \\
\chi\left(H_{1}\right) P_{1}^{\perp}\left(z-A_{1} / R\right)^{-1} \theta\left(H_{1}\right) P_{1}^{\perp} d z \wedge d \bar{z} \\
+P_{1}^{\perp} \theta\left(H_{1}\right)\left\langle A_{1} / R\right\rangle^{-s} R^{-2} B_{1}\left\langle A_{1} / R\right\rangle^{-s} \theta\left(H_{1}\right) P_{1}^{\perp} .
\end{gathered}
$$

Let $\varepsilon:=\rho_{0} / 2$. Using $(4.9)$, notice that

$$
\begin{aligned}
G & :=P_{1}^{\perp} \chi\left(H_{1}\right)\left[H_{1} \tau\left(H_{1}\right), i A_{1} / R\right]_{\circ} \chi\left(H_{1}\right) P_{1}^{\perp}=P_{1}^{\perp} \chi\left(H_{1}\right)\left[H_{1}, i A_{1} / R\right]_{\circ} \chi\left(H_{1}\right) P_{1}^{\perp} \\
& =P_{1}^{\perp} \chi\left(H_{1}\right)\left[H_{1}, i A_{1} / R\right]_{\circ} \chi\left(H_{0}\right) P_{1}^{\perp}+P_{1}^{\perp} \chi\left(H_{1}\right) K_{1} R^{-1} B_{2}\left\langle A_{1} / R\right\rangle^{-\varepsilon} P_{1}^{\perp}
\end{aligned}
$$


where the operator $K_{1}:=\tau\left(H_{1}\right)\left[H_{1}, i A_{1}\right]_{\circ}\left(\chi\left(H_{1}\right)-\chi\left(H_{0}\right)\right)\left\langle A_{1}\right\rangle^{\varepsilon}$ is compact and $B_{2}:=\left\langle A_{1} / R\right\rangle^{\varepsilon}\left\langle A_{1}\right\rangle^{-\varepsilon}$ is uniformly bounded. Similarly, there is $K_{2}$ compact so that

$$
\begin{aligned}
G= & P_{1}^{\perp} \chi\left(H_{0}\right)\left[H_{1}, i A_{1} / R\right]_{\circ} \chi\left(H_{0}\right) P_{1}^{\perp}+P_{1}^{\perp} \chi\left(H_{1}\right) K_{1} R^{-1} B_{2}\left\langle A_{1} / R\right\rangle^{-\varepsilon} P_{1}^{\perp} \\
& +P_{1}^{\perp}\left\langle A_{1} / R\right\rangle^{-\varepsilon} B_{2} K_{2} R^{-1} \chi\left(H_{0}\right) P_{1}^{\perp} .
\end{aligned}
$$

We focus on the potential contribution in $G$. Choosing $\tau$ appropriately and using Assumption 4.13, we claim that there exist a compact operator $K_{3}$ and an uniformly bounded operator $B_{3}$ such that

$$
\chi\left(H_{0}\right)\left[W+V, i A_{1} / R\right]_{\circ} \chi\left(H_{0}\right)=R^{-1} \chi\left(H_{0}\right) K_{3} B_{3}\left\langle A_{1} / R\right\rangle^{-\varepsilon} \chi\left(H_{0}\right) .
$$

By Lemma 4.3, we take the support of $\tau$ small enough to ensure the compactness of $\tau\left(H_{0}\right) W_{1} \tau\left(H_{0}\right)\left\langle A_{1}\right\rangle^{\varepsilon}$. By writing

$$
\left(W+x \cdot \nabla V_{\mathrm{lr}}\right) \tau\left(H_{0}\right)\left\langle A_{1}\right\rangle^{\varepsilon}=\left(W+x \cdot \nabla V_{\mathrm{lr}}\right)\langle x\rangle^{\rho_{0}} \cdot b^{w} \cdot\langle x\rangle^{-\varepsilon}\left\langle D_{x}\right\rangle^{-\varepsilon}\left\langle A_{1}\right\rangle^{\varepsilon},
$$

with $b \in \mathcal{S}\left(\langle x\rangle^{-\varepsilon}\langle\xi\rangle^{-1}, g\right), \tau\left(H_{0}\right)\left[W+V_{\mathrm{lr}}, i A_{1}\right]_{\circ} \tau\left(H_{0}\right)\left\langle A_{1}\right\rangle^{\varepsilon}$ is compact by $(2.15)$ and Lemma C.1. Similarly, we prove the compactness of $\tau\left(H_{0}\right)\left[V_{\mathrm{sr}}, i A_{1}\right]_{\circ} \tau\left(H_{0}\right)\left\langle A_{1}\right\rangle^{\varepsilon}$, making use of the fact that, by (4.5), $\left\langle D_{s}\right\rangle^{-1}\left[V_{\mathrm{sr}}, i A_{1}\right]_{\circ}\langle x\rangle^{\rho_{0}}\left\langle D_{s}\right\rangle^{-1}$ extends to a bounded operator. This yields (4.13).

Taking advantage of $\left[H_{0}, i A_{1}\right]_{\circ}=2 H_{0}$, of (4.13), and of (4.12), we rewrite (4.11):

$$
\begin{gathered}
F=\frac{i}{2 \pi} \int_{\mathbb{C}} \partial_{\bar{z}} \psi^{\mathbb{C}}(z) P_{1}^{\perp} \theta\left(H_{1}\right)\left(z-A_{1} / R\right)^{-1} P_{1}^{\perp} 2 R^{-1} H_{0} \chi^{2}\left(H_{0}\right) \\
P_{1}^{\perp}\left(z-A_{1} / R\right)^{-1} \theta\left(H_{1}\right) P_{1}^{\perp} d z \wedge d \bar{z} \\
+P_{1}^{\perp} \theta\left(H_{1}\right)\left\langle A_{1} / R\right\rangle^{-s}\left(R^{-2} B_{1}+R^{-1} K_{4}\right)\left\langle A_{1} / R\right\rangle^{-s} \theta\left(H_{1}\right) P_{1}^{\perp}
\end{gathered}
$$

with compact $K_{4}$ such that, for some $c_{1}>0$,

$$
\left\|K_{4}\right\| \leq c_{1}\left(\left\|P_{1}^{\perp} \chi\left(H_{1}\right) K_{1}\right\|+\left\|K_{2} \chi\left(H_{0}\right)\right\|+\left\|\chi\left(H_{0}\right) K_{3}\right\|\right) .
$$

Next we commute $\left(z-A_{1} / R\right)^{-1}$ with $P_{1}^{\perp} 2 R^{-1} H_{0} \chi^{2}\left(H_{0}\right) P_{1}^{\perp}$. Recalling (2.6) with $k=1$ and (4.10), there are $B_{4}$ and $B_{5}$, uniformly bounded, such that

$$
\begin{aligned}
& F=P_{1}^{\perp} \theta\left(H_{1}\right) \psi^{\prime}\left(A_{1} / R\right) P_{1}^{\perp} 2 R^{-1} H_{0} \chi^{2}\left(H_{0}\right) P_{1}^{\perp} \theta\left(H_{1}\right) P_{1}^{\perp} \\
&+P_{1}^{\perp} \theta\left(H_{1}\right)\left\langle A_{1} / R\right\rangle^{-s}\left(R^{-2} B_{4}+R^{-1} K_{4}\right)\left\langle A_{1} / R\right\rangle^{-s} \theta\left(H_{1}\right) P_{1}^{\perp} \\
&=P_{1}^{\perp} \theta\left(H_{1}\right)\left\langle A_{1} / R\right\rangle^{-s} 2 R^{-1} H_{0} \chi^{2}\left(H_{0}\right)\left\langle A_{1} / R\right\rangle^{-s} \theta\left(H_{1}\right) P_{1}^{\perp} \\
&+P_{1}^{\perp} \theta\left(H_{1}\right)\left\langle A_{1} / R\right\rangle^{-s}\left(R^{-2} B_{5}+R^{-1} K_{4}\right)\left\langle A_{1} / R\right\rangle^{-s} \theta\left(H_{1}\right) P_{1}^{\perp} \\
& \geq 2 R^{-1} c_{2} P_{1}^{\perp} \theta\left(H_{1}\right)\left\langle A_{1} / R\right\rangle^{-s} \chi^{2}\left(H_{0}\right)\left\langle A_{1} / R\right\rangle^{-s} \theta\left(H_{1}\right) P_{1}^{\perp} \\
& \quad+P_{1}^{\perp} \theta\left(H_{1}\right)\left\langle A_{1} / R\right\rangle^{-s}\left(R^{-2} B_{5}+R^{-1} K_{4}\right)\left\langle A_{1} / R\right\rangle^{-s} \theta\left(H_{1}\right) P_{1}^{\perp}
\end{aligned}
$$

where $c_{2}>0$ is the infimum of $\mathcal{I}$. Finally, since $K_{5}:=\chi^{2}\left(H_{0}\right)-\chi^{2}\left(H_{1}\right)$ is compact by (4.9), we find an uniformly bounded $B_{6}$, such that

$$
\begin{gathered}
F \geq 2 R^{-1} c_{2} P_{1}^{\perp} \theta\left(H_{1}\right)\left\langle A_{1} / R\right\rangle^{-s} \chi^{2}\left(H_{1}\right)\left\langle A_{1} / R\right\rangle^{-s} \theta\left(H_{1}\right) P_{1}^{\perp} \\
+P_{1}^{\perp} \theta\left(H_{1}\right)\left\langle A_{1} / R\right\rangle^{-s}\left(R^{-2} B_{5}+R^{-1} K_{4}+R^{-1} K_{5}\right)\left\langle A_{1} / R\right\rangle^{-s} \theta\left(H_{1}\right) P_{1}^{\perp} \\
\geq 2 R^{-1} c_{2} P_{1}^{\perp} \theta\left(H_{1}\right)\left\langle A_{1} / R\right\rangle^{-2 s} \theta\left(H_{1}\right) P_{1}^{\perp}+P_{1}^{\perp} \theta\left(H_{1}\right)\left\langle A_{1} / R\right\rangle^{-s} . \\
\left(R^{-2} B_{6}+R^{-1} K_{4}+R^{-1} K_{5} \chi\left(H_{1}\right) P_{1}^{\perp}\right)\left\langle A_{1} / R\right\rangle^{-s} \theta\left(H_{1}\right) P_{1}^{\perp} .
\end{gathered}
$$

To conclude, using (4.15), we decrease the support of $\chi$ to ensure that $\left\|K_{4}\right\|+$ $\left\|K_{5} \chi\left(H_{1}\right) P_{1}^{\perp}\right\|<c_{2}$. Subsequently, we choose $R>1$ large enough to guarantee 
$F \geq R^{-1} c_{2} P_{1}^{\perp} \theta\left(H_{1}\right)\left\langle A_{1} / R\right\rangle^{-2 s} \theta\left(H_{1}\right) P_{1}^{\perp}$. Letting act the projector $E_{\mathcal{I}}\left(H_{1}\right)$ on both sides of this inequality and recalling the definition of $F$, we get the projected, weighted Mourre estimate (3.5) with $H=H_{1}, P=P_{1}, B=\psi\left(A_{1} / R\right)$, and $C=$ $\sqrt{c_{2} / R}\left\langle A_{1} / R\right\rangle^{-s}$. By Theorem 3.4, we obtain the result.

\section{Usual Mourre theory.}

In this section, we explain why the usual Mourre theory with conjugate operator $A_{1}$ cannot be applied to $H_{1}$, the considered Schrödinger operator with oscillating potential. We have proved that $H_{1} \in \mathcal{C}^{1}\left(A_{1}\right)$ and established a Mourre estimate for $H_{1}$ w.r.t. $A_{1}$, see Propositions 4.2 and 4.8. However, in order to apply the standard Mourre theory, one has to prove that $H_{1}$ is in a better class of regularity w.r.t. $A_{1}$. In this section, we prove that this is not the case. If one replaces $A_{1}$ by some natural variants, we explain in Remark 5.6 below that the required regularity is not available. On the other hand, a consequence of Theorem 4.15 is that under the Assumption 4.13, the operator $H_{1}$ has no singular continuous spectrum. By abstract means, see [ABG, Proposition 7.2.14], there exists a conjugate operator $\tilde{A}$, such that $H_{1} \in \mathcal{C}^{\infty}(\tilde{A})$ and such that a strict Mourre estimate holds true for $H_{1}$, w.r.t. $\tilde{A}$, on every interval that contains neither an eigenvalue nor $\left\{0, k^{2} / 4\right\}$. It seems very difficult to find explicitly $\tilde{A}$.

We first continue the description of different classes of regularity appearing in the Mourre theory that we began in Subsection 2.1. We refer again to [ABG, GGM1, GGé] for more details. Recall that a self-adjoint operator $H$ belongs to the class $\mathcal{C}^{1}(A)$ if, for some (hence for all) $z \notin \sigma(H)$, the bounded operator $(H-z)^{-1}$ belongs to $\mathcal{C}^{1}(A)$. Lemma 6.2.9 and Theorem 6.2.10 in [ABG] gives the following characterization of this regularity:

Theorem 5.1. ([ABG]) Let $A$ and $H$ be two self-adjoint operators in the Hilbert space $\mathscr{H}$. For $z \notin \sigma(H)$, set $R(z):=(H-z)^{-1}$. The following points are equivalent:

(1) $H \in \mathcal{C}^{1}(A)$.

(2) For one (then for all) $z \notin \sigma(H)$, there is a finite $c$ such that

$$
|\langle A f, R(z) f\rangle-\langle R(\bar{z}) f, A f\rangle| \leq c\|f\|^{2} \text {, for all } f \in \mathcal{D}(A) \text {. }
$$

(3) a. There is a finite $c$ such that for all $f \in \mathcal{D}(A) \cap \mathcal{D}(H)$ :

$$
|\langle A f, H f\rangle-\langle H f, A f\rangle| \leq c\left(\|H f\|^{2}+\|f\|^{2}\right) .
$$

b. The set $\{f \in \mathcal{D}(A) ; R(z) f \in \mathcal{D}(A)$ and $R(\bar{z}) f \in \mathcal{D}(A)\}$ is a core for $A$, for some (then for all) $z \notin \sigma(H)$.

Note that the condition (3.b) could be uneasy to check, see [GGé]. We mention [GM][Lemma A.2] to overcome this subtlety. Note that (5.1) yields that the commutator $[A, R(z)]$ extends to a bounded operator, in the form sense. We shall denote the extension by $[A, R(z)]_{\circ}$. In the same way, from $(5.2)$, the commutator $[H, A]$ extends to a unique element of $\mathcal{B}\left(\mathcal{D}(H), \mathcal{D}(H)^{*}\right)$ denoted by $[H, A]_{\circ}$. Moreover, if $H \in \mathcal{C}^{1}(A)$ and $z \notin \sigma(H)$,

$$
\left[A,(H-z)^{-1}\right]_{\circ}=\underbrace{(H-z)^{-1}}_{\mathscr{H} \leftarrow \mathcal{D}(H)^{*}} \underbrace{[H, A]_{0}}_{\mathcal{D}(H)^{*} \leftarrow \mathcal{D}(H)} \underbrace{(H-z)^{-1}}_{\mathcal{D}(H) \leftarrow \mathscr{H}} .
$$


Here we use the Riesz lemma to identify $\mathscr{H}$ with its anti-dual $\mathscr{H}^{*}$. It turns out that an easier characterization is available if the domain of $H$ is conserved under the action of the unitary group generated by $A$.

Theorem 5.2. ([ABG, p. 258]) Let $A$ and $H$ be two self-adjoint operators in the Hilbert space $\mathscr{H}$ such that $e^{i t A} \mathcal{D}(H) \subset \mathcal{D}(H)$, for all $t \in \mathbb{R}$. Then $H \in \mathcal{C}^{1}(A)$ if and only if (5.2) holds true.

Remark 5.3. Some arguments used in the proof of Proposition 4.2 may be performed in an abstract way. Take a Hilbert space $\mathscr{G}$, such that $\mathscr{G} \hookrightarrow \mathscr{H}$ with a continuous, dense embedding and such that the $C_{0}$-group $\left\{e^{i t A}\right\}_{t \in \mathbb{R}}$ stabilizes $\mathscr{G}$ (hence also $\mathscr{G}^{*}$ by duality). Let $T \in \mathcal{B}\left(\mathscr{G}, \mathscr{G}^{*}\right)$. We say that $T \in \mathcal{C}^{1}\left(A ; \mathscr{G}, \mathscr{G}^{*}\right)$ if the strong limit of $t \mapsto t^{-1}\left(e^{i t A} T e^{-i t A}-T\right)$ exists in $\mathcal{B}\left(\mathscr{G}, \mathscr{G}^{*}\right)$, as $t$ goes to 0 . The limit is denoted by $[T, i A]_{\circ}$. Assuming the invariance of $\mathcal{D}(H)$ under the $C_{0}$-group $\left\{e^{i t A}\right\}_{t \in \mathbb{R}}$ and taking a $\mathscr{G}$ with continuous, dense embeddings $\mathcal{D}(H) \hookrightarrow \mathscr{G} \hookrightarrow \mathscr{H}$, then $\left\{e^{i t A}\right\}_{t \in \mathbb{R}}$ stabilizes $\mathscr{G}$ by interpolation. If $T \in \mathcal{C}^{1}\left(A ; \mathscr{G}, \mathscr{G}^{*}\right)$ then $T \in \mathcal{C}^{1}\left(A ; \mathcal{D}(H), \mathcal{D}(H)^{*}\right)$. If $H \in \mathcal{C}^{1}\left(A ; \mathcal{D}(H), \mathcal{D}(H)^{*}\right),[H, i A]_{\circ}$ coincide with the previous definition. One can reformulate Theorem 5.2 as follows: $H \in \mathcal{C}^{1}\left(A ; \mathcal{D}(H), \mathcal{D}(H)^{*}\right)$ if and only if $H \in \mathcal{C}^{1}(A)$.

We need to introduce others classes inside $\mathcal{C}^{1}(A)$. Let $T \in \mathcal{B}(\mathscr{H})$. We say that $T \in \mathcal{C}^{1, u}(A)$ if the map $\mathbb{R} \ni t \mapsto e^{i t A} T e^{-i t A} \in \mathcal{B}(\mathscr{H})$ has the usual $\mathcal{C}^{1}$ regularity. We say that $T \in \mathcal{C}^{1,1}(A)$ if

$$
\int_{0}^{1}\left\|\left[\left[T, e^{i t A}\right], e^{i t A}\right]\right\| t^{-2} d t<\infty
$$

We say that $T \in \mathcal{C}^{1+0}(A)$ if $T \in \mathcal{C}^{1}(A)$ and

$$
\int_{0}^{1}\left\|e^{i t A}[T, A] e^{-i t A}\right\| t^{-1} d t<\infty .
$$

Thanks to $[\mathrm{ABG}$, p. 205], it turns out that

$$
\mathcal{C}^{2}(A) \subset \mathcal{C}^{1+0}(A) \subset \mathcal{C}^{1,1}(A) \subset \mathcal{C}^{1, u}(A) \subset \mathcal{C}^{1}(A) .
$$

Given a self-adjoint operator $H$ and an open interval $\mathcal{I}$ of $\mathbb{R}$, we consider the corresponding local classes defined by: $H \in \mathcal{C}_{\mathcal{I}}^{[\cdot]}(A)$ if, for all $\varphi \in \mathcal{C}_{c}^{\infty}(\mathcal{I}), \varphi(H) \in \mathcal{C}^{[\cdot]}(A)$. We say that $H \in \mathcal{C}^{[\cdot]}(A)$ if, for some $z \notin \sigma(H), R(z) \in \mathcal{C}^{[\cdot]}(A)$. Proposition 2.2 also works for the new classes: for all open interval $\mathcal{I}$ of $\mathbb{R}$ and all $\varphi \in \mathcal{C}_{c}^{\infty}(\mathcal{I})$,

$$
H \in \mathcal{C}^{[\cdot]}(A) \quad \Longrightarrow \quad \varphi(H) \in \mathcal{C}_{\mathcal{I}}^{[\cdot]}(A) \text {. }
$$

In $[\mathrm{ABG}]$, the LAP is obtained for $H \in \mathcal{C}^{1,1}(A)$ (see p. 308 and p. 317) and this class is shown to be optimal among the global classes (see the end of Section 7.B). In [Sa], for $H \in \mathcal{C}_{\mathcal{I}}^{1+0}(A)$, the LAP is obtained on compact sub-interval of $\mathcal{I}$. It is expected that the class $\mathcal{C}_{\mathcal{I}}^{1,1}(A)$ is sufficient. Section 7.B in $[\mathrm{ABG}]$ again shows that one cannot use in general a bigger local class to get the LAP.

Now we explore the regularity properties of $H_{1}$ under Assumption 4.6. From Proposition 4.2 , we know that $H_{1} \in \mathcal{C}^{1}\left(A_{1}\right)$. If $H_{1}$ would belong to $\mathcal{C}^{1,1}\left(A_{1}\right)$ then, by $(5.6)$ and (5.7), $H_{1}$ would belong to $\mathcal{C}_{\mathcal{I}}^{1, u}\left(A_{1}\right)$ for any open interval $\left.\mathcal{I} \subset\right] 0 ;+\infty\left[\right.$. If $H_{1}$ would belong to $\mathcal{C}_{\mathcal{I}}^{1+0}\left(A_{1}\right)$ or even to $\mathcal{C}_{\mathcal{I}}^{1,1}\left(A_{1}\right)$, for some open interval $\left.\mathcal{I} \subset\right] 0 ;+\infty[$, then $H_{1}$ would belong to $\mathcal{C}_{\mathcal{I}}^{1, u}\left(A_{1}\right)$ by $(5.6)$. In both cases, this would contradict: 
Proposition 5.4. Under Assumption 4.6, for any open sub-interval $\mathcal{I}$ of $] 0 ;+\infty[$, $H_{1} \notin \mathcal{C}_{\mathcal{I}}^{1, u}\left(A_{1}\right)$.

Proof. Take such an interval $\mathcal{I}$ and $\varphi \in \mathcal{C}_{c}^{\infty}(\mathcal{I})$. By Proposition 4.2, (5.6), and (5.7), $\varphi\left(H_{0}\right) \in \mathcal{C}^{1, u}\left(A_{1}\right)$. Assume that $\varphi\left(H_{1}\right) \in \mathcal{C}^{1, u}\left(A_{1}\right)$. Then $K:=\varphi\left(H_{1}\right)-\varphi\left(H_{0}\right) \in$ $\mathcal{C}^{1, u}\left(A_{1}\right)$ and $K$ is a compact operator on $\mathrm{L}^{2}\left(\mathbb{R}_{x}^{d}\right)$, thanks to Lemma 4.7. Thus

$$
\left[K, i A_{1}\right]_{\circ}=\lim _{t \rightarrow 0} t^{-1}\left(e^{-i t A_{1}} K e^{i t A_{1}}-K\right)
$$

in $\mathcal{B}\left(\mathrm{L}^{2}\left(\mathbb{R}_{x}^{d}\right)\right)$ and $\left[K, i A_{1}\right]_{\circ}$ is also compact. So is $B\left[K, i A_{1}\right]_{\circ} B^{\prime}$, for any $B, B^{\prime} \in$ $\mathcal{B}\left(\mathrm{L}^{2}\left(\mathbb{R}_{x}^{d}\right)\right)$. This contradicts Lemma 5.5 below.

Lemma 5.5. Assume Assumption 4.6. For any open interval $\mathcal{I} \subset] 0 ;+\infty[$, there exist a function $\varphi \in \mathcal{C}_{c}^{\infty}(\mathcal{I})$ and bounded operators $B, B^{\prime}$ on $\mathrm{L}^{2}\left(\mathbb{R}_{x}^{d}\right)$ such that $B\left[\varphi\left(H_{1}\right)-\varphi\left(H_{0}\right), i A_{1}\right]_{\circ} B^{\prime}$ is not compact on $\mathrm{L}^{2}\left(\mathbb{R}_{x}^{d}\right)$.

We refer to Appendix $\mathrm{D}$ for a proof of this Lemma for $d=1$, which does not rely on pseudodifferential calculus.

Proof of Lemma 5.5. In the sequel, for $C, D \in \mathcal{B}\left(\mathrm{L}^{2}\left(\mathbb{R}_{x}^{d}\right)\right)$, we write $C \simeq D$ if $C-D$ is compact on $\mathrm{L}^{2}\left(\mathbb{R}_{x}^{d}\right)$. By Proposition $4.2, H_{1}, H_{0} \in \mathcal{C}^{1}(A)$. Then $B_{1}:=$ $\left[\varphi\left(H_{1}\right)-\varphi\left(H_{0}\right), i A_{1}\right]_{\circ}$ is bounded. Furthermore, thanks to $(2.9),(2.10)$, and by the resolvent formula, with a norm convergent integral,

$$
B_{1}=\frac{i}{2 \pi} \int_{\mathbb{C}} \partial_{\bar{z}} \varphi^{\mathbb{C}}(z)\left[\left(z-H_{1}\right)^{-1}(W+V)\left(z-H_{0}\right)^{-1}, i A_{1}\right]_{\circ} d z \wedge d \bar{z}
$$

We recall that given a continuous function $F: \mathbb{R}^{d} \rightarrow \mathbb{C}$, that tends to 0 at infinity, the multiplication by $F$ is compact from $\mathrm{H}^{s}\left(\mathbb{R}_{x}^{d}\right)$ to $L^{2}\left(\mathbb{R}_{x}^{d}\right)$, for all $s>0$. Using again Proposition 4.2 and expanding the commutator, using the computation of $\left[W, i A_{1}\right]_{\circ}$ (see just before (4.4)) and the resolvent formula again, it yields:

$$
\begin{aligned}
B_{1} & \simeq \frac{i}{2 \pi} \int_{\mathbb{C}} \partial_{\bar{z}} \varphi^{\mathbb{C}}(z)\left(z-H_{1}\right)^{-1}\left[W+V, i A_{1}\right]_{\circ}\left(z-H_{0}\right)^{-1} d z \wedge d \bar{z}, \\
& \simeq \frac{-i}{2 \pi} \int_{\mathbb{C}} \partial_{\bar{z}} \varphi^{\mathbb{C}}(z)\left(z-H_{1}\right)^{-1} W_{1}\left(z-H_{0}\right)^{-1} d z \wedge d \bar{z}, \\
& \simeq \frac{-i}{2 \pi} \int_{\mathbb{C}} \partial_{\bar{z}} \varphi^{\mathbb{C}}(z)\left(z-H_{0}\right)^{-1} W_{1}\left(z-H_{0}\right)^{-1} d z \wedge d \bar{z}, \\
& \simeq \frac{-i}{2 \pi} \int_{\mathbb{C}} \partial_{\bar{z}} \varphi^{\mathbb{C}}(z)\left(z-H_{0}\right)^{-1} \chi_{1} W_{1}\left(z-H_{0}\right)^{-1} d z \wedge d \bar{z},
\end{aligned}
$$

with $\chi_{1} \in \mathcal{C}^{\infty}\left(\mathbb{R}^{d}\right), \chi_{1}=0$ near 0 , and $\chi_{1}=1$ near infinity.

At this point, we use pseudodifferential techniques and, in particular, Appendix A. For $x \in \mathbb{R}^{d}$, let $e_{ \pm}(x)=\chi_{1}(x) e^{ \pm i k|x|}$. By $(4.4),\left(\chi_{1} W_{1}\right)(x)=k q 2^{-1}\left(e_{+}(x)+e_{-}(x)\right)$. Now we apply Proposition A.1 to $a(x, \xi)=|\xi|^{2} \in \mathcal{S}\left(\langle\xi\rangle^{2}, g\right)$. By its proof, $a_{ \pm}$can be chosen real and $a_{ \pm}^{w}$ is self-adjoint. Using the resolvents of $a_{ \pm}^{w}$ and $a^{w}$,

$$
\begin{aligned}
e_{ \pm}\left(z-H_{0}\right)^{-1}=e_{ \pm} & \left(z-a^{w}\right)^{-1}=\left(z-a_{ \pm}^{w}\right)^{-1} e_{ \pm} \\
& +\left(z-a_{ \pm}^{w}\right)^{-1}\left(e_{ \pm} b_{ \pm}^{w}+c_{ \pm}^{w} e_{ \pm}\right)\left(z-a^{w}\right)^{-1}
\end{aligned}
$$




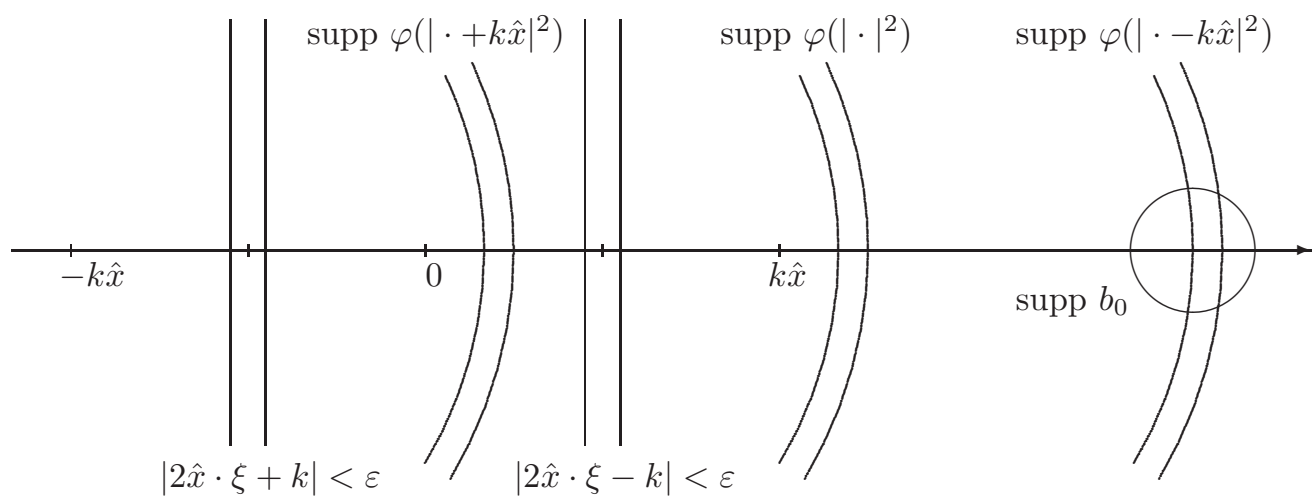

FiguRE 3. $\operatorname{supp} b_{0}$

for all $z \notin \mathbb{R}$. We obtain from (5.10), (5.11), and Proposition A.1:

$$
B_{1} \simeq-\sum_{\sigma= \pm} \frac{i k q}{4 \pi} \int_{\mathbb{C}} \partial_{\bar{z}} \varphi^{\mathbb{C}}(z)\left(z-H_{0}\right)^{-1}\left(z-a_{\sigma}^{w}\right)^{-1} e_{\sigma} d z \wedge d \bar{z} .
$$

According to [Bo1] (see Appendix B), $\left(z-H_{0}\right)^{-1}=p_{z}^{w}$ and $\left(z-a_{\sigma}^{w}\right)^{-1}=p_{\sigma, z}^{w}$ where the symbols $p_{z}, p_{\sigma, z}$ belong to $\mathcal{S}\left(\langle\xi\rangle^{-2}, g\right)$ and satisfy (B.3) with $m=\langle\xi\rangle^{2}$. Using the continuity of the map $\mathcal{S}\left(\langle\xi\rangle^{-2}, g\right)^{2} \ni(r, t) \mapsto r \# t-r t \in \mathcal{S}\left(h\langle\xi\rangle^{-2}, g\right)$, we can find, for all $\ell \in \mathbb{N}, C_{\ell}>0$ and $\mathbb{N}_{\ell} \in \mathbb{N}$ such that

$$
\left\|p_{z} \# p_{\sigma, z}-p_{z} p_{\sigma, z}\right\|_{\ell, \mathcal{S}\left(h\langle\xi\rangle^{-4}, g\right)} \leq C_{\ell}\langle z\rangle^{N_{\ell}+1}|\operatorname{Im}(z)|^{-N_{\ell}-1} .
$$

Using (2.3), (2.4), and (2.5), we see that, for $\sigma \in\{+;-\}$,

$$
\int_{\mathbb{C}} \partial_{\bar{z}} \varphi^{\mathbb{C}}(z)\left(p_{z} \# p_{\sigma, z}-p_{z} p_{\sigma, z}\right) d z \wedge d \bar{z}
$$

converges in $\mathcal{S}\left(h\langle\xi\rangle^{-4}, g\right)=\mathcal{S}\left(\langle x\rangle^{-1}\langle\xi\rangle^{-5}, g\right)$. Thanks to (2.15),

$$
B_{1} \simeq-\sum_{\sigma= \pm} \frac{i k q}{4 \pi}\left(\int_{\mathbb{C}} \partial_{\bar{z}} \varphi^{\mathbb{C}}(z)\left(z-|\xi|^{2}\right)^{-1}\left(z-a_{\sigma}(x, \xi)\right)^{-1} d z \wedge d \bar{z}\right)^{w} e_{\sigma}
$$

We take $b \in \mathcal{S}(1, g)$ such that $b \chi_{1}=b$. By the previous arguments,

$$
\begin{gathered}
b^{w} B_{1} \simeq-\sum_{\sigma= \pm}\left(\int_{\mathbb{C}} \partial_{\bar{z}} \varphi^{\mathbb{C}}(z) b(x, \xi)\left(z-|\xi|^{2}\right)^{-1}\left(z-a_{\sigma}(x, \xi)\right)^{-1} d z \wedge d \bar{z}\right)^{w} \\
\cdot i k q(4 \pi)^{-1} e^{i \sigma k|x|}
\end{gathered}
$$

Now we choose $\varphi$ with a small enough support near some $\lambda \in \mathcal{I}$ and $b \in \mathcal{S}(1, g)$ such that $b(x, \xi)=\chi_{4}(x) b_{0}(\hat{x}, \xi), \chi_{4} \in \mathcal{C}^{\infty}\left(\mathbb{R}^{d}\right)$ with $\chi_{4}=0$ near 0 and $\chi_{4}=1$ near infinity, $\varphi\left(|\xi|^{2}\right) b_{0}(\hat{x}, \xi)=0=\varphi\left(|\xi+k \hat{x}|^{2}\right) b_{0}(\hat{x}, \xi), b_{0}=0$ near $\xi \cdot \hat{x}= \pm k / 2$, and such that $\varphi\left(|\xi-k \hat{x}|^{2}\right) b_{0}(\hat{x}, \xi)$ is nonzero, see Figure 3 . In the last requirement, we use the fact that $\mathcal{I} \subset] 0 ;+\infty\left[\right.$. Note that, on the support of $b_{0}(\hat{x}, \xi)$ and for $|x|$ large enough, $b_{1}(x, \xi):=|\xi|^{2}-|\xi+\sigma k \hat{x}|^{2}$ does not vanish, for $\sigma \in\{+;-\}$. Thus,

$$
\left(z-|\xi|^{2}\right)^{-1}\left(z-a_{\sigma}(x, \xi)\right)^{-1}=\left(b_{1}(x, \xi)\right)^{-1}\left(\left(z-|\xi|^{2}\right)^{-1}-\left(z-a_{\sigma}(x, \xi)\right)^{-1}\right),
$$


in this region. Inserting this in (5.13) and using the support properties of $b$ and $\varphi$,

$$
\begin{aligned}
b^{w} B_{1} & \simeq-k q 2^{-1} \sum_{\sigma= \pm}\left(b(x, \xi)\left(b_{1}(x, \xi)\right)^{-1}\left(\varphi\left(|\xi|^{2}\right)-\varphi\left(|\xi+\sigma k \hat{x}|^{2}\right)\right)\right)^{w} e^{i \sigma k|x|} \\
& \simeq k q 2^{-1}\left(b(x, \xi)\left(b_{1}(x, \xi)\right)^{-1} \varphi\left(|\xi-k \hat{x}|^{2}\right)\right)^{w} e^{-i k|x|} .
\end{aligned}
$$

Setting $B=b^{w}$ and $B^{\prime}=e^{i k|x|}, B B_{1} B^{\prime} \simeq c^{w}$ with an explicit $c \in \mathcal{S}(1, g)$ that does not tend to 0 at infinity. By (2.15), neither $c^{w}$ nor $B B_{1} B^{\prime}$ is compact.

Remark 5.6. As alternative to $A_{1}$, it is natural to try $\hat{A}_{1}=(\tau(\xi) \rho(x) x \cdot \xi)^{w}$, where $1-\rho \in \mathcal{C}_{c}^{\infty}\left(\mathbb{R}^{d}\right)$ and $\tau \in S(1, g)$ satisfies $\tau(\xi)=1$ if $|\xi|^{2} \in \mathcal{I}$. But Proposition 5.3 holds true with $A_{1}$ replaced by $\hat{A}_{1}$.

Let us sketch a justification of Remark 5.6. One can verify that $\varphi\left(H_{0}\right) \in \mathcal{C}^{2}\left(\hat{A}_{1}\right)$. We follow the proof of Lemma 5.5 and arrive at (5.10) where $\chi_{1} W_{1}$ is replaced by:

$$
-\sum_{\sigma= \pm} \frac{i k q}{4 \pi} \int_{\mathbb{C}} \partial_{\bar{z}} \varphi^{\mathbb{C}}(z)\left(z-H_{0}\right)^{-1} b_{\tau, \sigma}^{w} d z \wedge d \bar{z} e_{\sigma}
$$

with, for $\chi_{2}=0$ near 0 and $\chi_{2} \chi_{1}=\chi_{1}$,

$$
b_{\tau, \sigma}=\chi_{2}(x)(\tau(\xi) \hat{x} \cdot \xi-\tau(\xi-\sigma k \hat{x}) \hat{x} \cdot(\xi-\sigma k \hat{x})) .
$$

Since the $b_{\tau, \sigma}$ do not depend on $z$, we can estimate $p_{z} \# b_{\tau, \sigma} \# p_{\sigma, z}-b_{\tau, \sigma} p_{z} p_{\sigma, z}$ in a similar way as in (5.12) and get (5.13) with $b$ replaced by $b b_{\tau, \sigma}$. Following the last lines, we find that $B B_{1} B^{\prime} \simeq\left(b_{\tau,-} c\right)^{w}, b_{\tau,-} c \in \mathcal{S}(1, g)$, and $b_{\tau,-} c$ does not tend to zero at infinity. We arrive at the same conclusion as in Lemma 5.5.

\section{Appendix A. Oscillating terms.}

In our study of Schrödinger operator with a perturbed Wigner-Von Neumann potential (see Section 4 ), we need a good understanding of operator compositions like $a^{w} \chi_{1} W_{1}$, where $a \in \mathcal{S}(m, g), g$ and $m$ given by (2.12) and (2.13), $W_{1}$ given by (4.4), and where $\chi_{1} \in \mathcal{C}^{\infty}\left(\mathbb{R}^{d}\right)$ such that $\chi_{1}=0$ near 0 and $\chi_{1}=1$ near infinity. More precisely, we are looking for an explicit pseudodifferential operator $A$ such that $a^{w} \chi_{1} W_{1}=A+b_{1}^{w} B_{1}+B_{2} b_{2}^{w}$, with bounded operators $B_{1}, B_{2}$ and symbols $b_{1}, b_{2} \in \mathcal{S}\left(m\langle x\rangle^{-1}\langle\xi\rangle^{-1}, g_{0}\right)$ ( $g_{0}$ given in (2.12)). Although $a \in \mathcal{S}\left(m, g_{0}\right)$ and $\chi_{1} W_{1} \in \mathcal{S}\left(\langle x\rangle^{-1}, g_{0}\right)$, the symbolic calculus associated to $g_{0}$ is not well suited for our analysis, in particular to guarantee $b_{1}, b_{2} \in \mathcal{S}\left(m\langle x\rangle^{-1}\langle\xi\rangle^{-1}, g_{0}\right)$. It is better to work with $g$ with the drawback that $W_{1}$ does not belong the corresponding calculus. Taking into account the special form of $W_{1}$, we provide the previous decomposition with $b_{1}, b_{2} \in \mathcal{S}\left(m\langle x\rangle^{-1}\langle\xi\rangle^{-1}, g\right)$, using standard arguments of pseudodifferential calculus. In Appendix $\mathrm{D}$, we give a simpler result in dimension $d=1$ that essentially follows from facts used in $[\mathrm{FH}]$.

For $m$ of the form $(2.13)$, we denote by $\mathcal{S}\left(m\langle x\rangle^{-\infty}, g\right)$ the intersection of all classes $\mathcal{S}\left(m\langle x\rangle^{k}, g\right)$ for $k \in \mathbb{Z}$. We denote by $\mathcal{S}(-\infty, g)$ the intersection of all classes $\mathcal{S}(m, g)$ with $m$ satisfying (2.13). It suffices to study $a^{w} e_{ \pm}$where $e_{ \pm}(x)=\chi_{1}(x) e^{ \pm i k|x|}$. To this end, we shall use the oscillatory integrals defined in Theorem 7.8.2, p. 237, in [Hö1], which actually works for symbols in the classes $\mathcal{S}(m, g)$ we consider here. These oscillatory integrals can also be viewed as tempered distributions. Note that 
usual operations on integrals (like integration by parts or change of variable) are valid for oscillatory integrals.

Proposition A.1. Let $a \in \mathcal{S}(m, g)$ with $m$ and $g$ given by (2.13) and (2.12). Let $e_{ \pm}$be the functions defined just above. Then there exist symbols $a_{ \pm} \in \mathcal{S}(m, g), b_{ \pm} \in$ $\mathcal{S}\left(m\langle x\rangle^{-\infty}, g\right)$, and $c_{ \pm} \in \mathcal{S}(m h, g)$ (with $h$ defined in (2.14)), such that $e_{ \pm} a^{w}=$ $a_{ \pm}^{w} e_{ \pm}+e_{ \pm} b_{ \pm}^{w}+c_{ \pm}^{w} e_{ \pm}$and such that $a_{ \pm}(x, \xi)=a\left(x, \xi \mp k|x|^{-1} x\right)$, if $\chi_{1}(x) \neq 0$.

Proof. Let $\chi_{2}, \check{\chi}_{2} \in \mathcal{C}^{\infty}\left(\mathbb{R}^{d}\right)$ such that $\chi_{2}=0$ and $\check{\chi}_{2}=0$ near $0, \check{\chi}_{2} \chi_{1}=\chi_{1}$, and $\check{\chi}_{2}\left(1-\chi_{2}\right)=0$. Notice that $\chi_{2} \chi_{1}=\chi_{1}$. We write $e_{ \pm} a^{w}=e_{ \pm} a^{w}\left(\chi_{2}+1-\chi_{2}\right)=$ $e_{ \pm} a^{w} \chi_{2} e^{\mp i k|x|} e_{ \pm}+e_{ \pm} \check{\chi}_{2} a^{w}\left(1-\chi_{2}\right)$ and arrive at

$$
e_{ \pm} a^{w}=e_{ \pm} a^{w} \chi_{2} e^{\mp i k|x|} e_{ \pm}+e_{ \pm} b^{w}
$$

where $b:=\check{\chi}_{2} \# a \#\left(1-\chi_{2}\right) \in \mathcal{S}(-\infty, g)$, since $\check{\chi}_{2}\left(1-\chi_{2}\right)=0$. For $f \in \mathscr{S}\left(\mathbb{R}^{d}\right)$, the Schwartz space on $\mathbb{R}^{d}$, using an oscillatory integral in the $\xi$ variable,

$$
\begin{aligned}
f_{1}(x) & :=\left(e_{ \pm} a^{w} \chi_{2} e^{\mp i k|x|} f\right)(x) \\
& =(2 \pi)^{-d} \int e^{i\langle x-y, \xi\rangle} a((x+y) / 2 ; \xi) \chi_{1}(x) e^{ \pm i k|x|} \cdot \chi_{2}(y) e^{\mp i k|y|} f(y) d y d \xi \\
& =(2 \pi)^{-d} \int e^{i\langle x-y, \xi\rangle \pm i k(|x|-|y|)} a((x+y) / 2 ; \xi) \cdot \chi_{1}(x) \chi_{2}(y) f(y) d y d \xi
\end{aligned}
$$

We take $\varepsilon \in] 0 ; 1 / 4\left[\right.$ and $\tau \in \mathcal{C}_{c}^{\infty}(\mathbb{R})$ such that $\tau(t)=1$ if $|t| \leq 1-4 \varepsilon$ and $\tau(t)=0$ if $|t| \geq 1-2 \varepsilon$. We insert $\tau\left(|x-y|\langle x\rangle^{-1}\right)+1-\tau\left(|x-y|\langle x\rangle^{-1}\right)$ into the previous expression of $f_{1}$ and call $f_{2}$ (resp. $f_{3}$ ) the integral containing $\tau\left(|x-y|\langle x\rangle^{-1}\right.$ ) (resp. $1-\tau(\mid x-$ $\left.\left.y \mid\langle x\rangle^{-1}\right)\right)$. On the support of $\chi_{1}(x) \chi_{2}(y) \tau\left(|x-y|\langle x\rangle^{-1}\right),|x-y| \leq(1-2 \varepsilon)\langle x\rangle$. We can choose the support of $\chi_{1}$ such that, on the support of $\chi_{1}(x) \chi_{2}(y) \tau\left(|x-y|\langle x\rangle^{-1}\right)$, $|x-y| \leq(1-\varepsilon)|x|$. In particular, on this support, 0 does not belong the segment $[x ; y]$ and, for all $t \in[0 ; 1]$,

$$
u(t ; x, y):=|t x+(1-t) y| \geq|x|-(1-t)|y-x| \geq \varepsilon|x| .
$$

For $x \neq y,\left(L_{x, y, D_{\xi}}-1\right) e^{i\langle x-y, \xi\rangle \pm i k(|x|-|y|)}=0$ for $L_{x, y, D_{\xi}}=|x-y|^{-2}(x-y) \cdot D_{\xi}$. Thus, by integration by parts, for all $p \in \mathbb{N}$,

$$
\begin{aligned}
f_{3}(x) & =(2 \pi)^{-d} \int e^{i\langle x-y, \xi\rangle \pm i k(|x|-|y|)} \chi_{1}(x) \chi_{2}(y)\left(1-\tau\left(|x-y|\langle x\rangle^{-1}\right)\right) \\
\cdot & \cdot\left(L_{x, y, D_{\xi}}^{*}\right)^{p}(a((x+y) / 2 ; \xi)) f(y) d y d \xi \\
& =\left(b_{3}^{w} f\right)(x),
\end{aligned}
$$

with $b_{3} \in \mathcal{S}(-\infty, g)$ (cf., (8.1.8) in [Hö3]).

Lemma A.2. Take $x, y \in \mathbb{R}^{d}$ such that 0 does not belong the segment $[x ; y]$. Then,

$$
|x|-|y|=\langle v(1 / 2 ; x, y)+r(x, y), x-y\rangle
$$

where $v(t ; x, y)=(t x+(1-t) y) /|t x+(1-t) y|$ for $t \in[0 ; 1]$, and where

$$
r(x, y):=\int\left((1-t) \mathbb{1}_{[1 / 2 ; 1]}(t)-t \mathbb{1}_{[0 ; 1 / 2]}(t)\right) \partial_{t} v(t ; x, y) d t
$$

satisfies $|r(x, y)| \leq 2$. 
Proof. It suffices to use the Taylor expansion with integral rest for the function $u(\cdot ; x, y)$ defined in $($ A.2) between 0 and $1 / 2$ and between $1 / 2$ and 1 .

By Lemma A.2, we can rewrite $f_{2}(x)$ as

$$
\begin{gathered}
f_{2}(x)=(2 \pi)^{-d} \int e^{i\langle x-y, \xi \pm k(v(1 / 2 ; x, y)+r(x, y))\rangle} \chi_{1}(x) \chi_{2}(y) \tau\left(|x-y|\langle x\rangle^{-1}\right) \\
\cdot a((x+y) / 2 ; \xi) f(y) d y d \xi \\
=(2 \pi)^{-d} \int e^{i\langle x-y, \eta\rangle} \chi_{1}(x) \chi_{2}(y) \tau\left(|x-y|\langle x\rangle^{-1}\right) \\
\cdot a((x+y) / 2 ; \eta \mp k(v(1 / 2 ; x, y)+r(x, y))) f(y) d y d \eta
\end{gathered}
$$

after the change of variable $\eta=\xi \pm k(v(1 / 2 ; x, y)+r(x, y))$. Now we use a Taylor expansion of $a$ with integral rest in the $\xi$ variable:

$$
\begin{array}{r}
a((x+y) / 2 ; \eta \mp k(v(1 / 2 ; x, y)+r(x, y)))=a((x+y) / 2 ; \eta \mp k v(1 / 2 ; x, y)) \\
+\int_{0}^{1} d t\left\langle\nabla_{\xi} a((x+y) / 2 ; \eta \mp k(v(1 / 2 ; x, y)+\operatorname{tr}(x, y))), k r(x, y)\right\rangle
\end{array}
$$

According to this decomposition, we split $f_{2}(x)$ in $f_{4}(x)+f_{5}(x)$. Thanks to the bound (A.2) for $t=1 / 2$, we can find $\chi_{3} \in \mathcal{C}^{\infty}\left(\mathbb{R}^{d}\right)$ such that $\chi_{3}=0$ near 0 and

$$
\chi_{1}(x) \chi_{2}(y) \tau\left(|x-y|\langle x\rangle^{-1}\right)\left(1-\chi_{3}((x+y) / 2)\right)=0 .
$$

Setting $a_{ \pm}(x, \eta)=\chi_{3}(x) a(x, \eta \mp k \hat{x})$, we obtain that

$$
\begin{aligned}
f_{2}(x)=(2 \pi)^{-d} \int e^{i\langle x-y, \eta\rangle} \chi_{1}(x) \chi_{2}(y) \tau\left(|x-y|\langle x\rangle^{-1}\right) \\
\cdot a_{ \pm}((x+y) / 2 ; \eta) f(y) d y d \xi+f_{5}(x) \\
=\chi_{1}(x)\left(a_{ \pm}^{w} \chi_{2} f\right)(x)+f_{5}(x)=\left(a_{ \pm}^{w} f\right)(x)+\left(b_{2}^{w} f\right)(x)+f_{5}(x),
\end{aligned}
$$

with $b_{2} \in \mathcal{S}\left(m\langle x\rangle^{-\infty}, g\right)$. Since $\| \eta+k \hat{x}|-| \eta|| \leq k$, for all $x \in \mathbb{R}^{d} \backslash\{0\}$ and all $\eta \in \mathbb{R}^{d}$, a direct computation shows that $a_{ \pm} \in \mathcal{S}(m, g)$.

Now we study $f_{5}$. Given a vector $v \in \mathbb{R}^{d}$, let $A(v)=I-\langle v, \cdot\rangle v$ (where $I$ denotes the identity on $\left.\mathbb{R}^{d}\right)$. If 0 does not belong to the segment $[x ; y]$ in $\mathbb{R}^{d}, \partial_{t} v(t ; x, y)=$ $(u(t ; x, y))^{-1} A(v(t ; x, y)) \cdot(x-y)$, where $v(t ; x, y)$ (resp. $\left.u(t ; x, y)\right)$ is defined in Lemma A.2 (resp. (A.2)). Defining $\kappa(s):=(1-s) \mathbb{1}_{[1 / 2 ; 1]}(s)-s \mathbb{1}_{[0 ; 1 / 2](s)}$,

$$
\begin{array}{r}
f_{5}(x)=(2 \pi)^{-d} \int e^{i\langle x-y, \eta\rangle} \chi_{1}(x) \chi_{2}(y) \tau\left(|x-y|\langle x\rangle^{-1}\right) \int_{0}^{1} d t \\
\cdot\left\langle\nabla_{\xi} a((x+y) / 2 ; \eta \mp k(v(1 / 2 ; x, y)+\operatorname{tr}(x, y))),\right. \\
\left.\cdot k \int_{0}^{1} d s \kappa(s)(u(s ; x, y))^{-1} A(v(s ; x, y)) \cdot(x-y)\right\rangle \cdot f(y) d y d \xi,
\end{array}
$$

by (A.5). Denoting by $A(v)^{T}$ the transposed of the linear map $A(v)$ and setting $\eta_{t}=\eta \mp k(v(1 / 2 ; x, y)+\operatorname{tr}(x, y))$,

$$
\begin{aligned}
\left\langle\nabla_{\xi} a\left((x+y) / 2 ; \eta_{t}\right), A(v(s ; x, y))\right. & \cdot(x-y)\rangle \\
= & \left\langle A(v(s ; x, y))^{T} \nabla_{\xi} a\left((x+y) / 2 ; \eta_{t}\right),(x-y)\right\rangle .
\end{aligned}
$$


Integrating by parts in the $\eta$ variable,

$$
\begin{aligned}
f_{5}(x)=(2 \pi)^{-d} \int e^{i\langle x-y, \eta\rangle} \chi_{1}(x) \chi_{2}(y) \tau\left(|x-y|\langle x\rangle^{-1}\right) \int_{0}^{1} d t \int_{0}^{1} d s \\
\cdot\left(i\left\langle A(v(s ; x, y)) \nabla_{\xi}, \nabla_{\xi}\right\rangle a\right)\left((x+y) / 2 ; \eta_{t}\right) \\
\cdot k \kappa(s)(u(s ; x, y))^{-1} f(y) d y d \xi
\end{aligned}
$$

Writing $f(y)=(2 \pi)^{-d} \int e^{i\langle y, \xi\rangle}(\mathcal{F} f)(\xi) d \xi, \mathcal{F} f$ being the Fourier transform of $f$,

$$
f_{5}(x)=(2 \pi)^{-d} \int e^{i\langle x, \xi\rangle} c_{0}(x, \xi)(\mathcal{F} f)(\xi) d \xi=\left(\mathrm{Op} c_{0} f\right)(x)
$$

where $c_{0}$ is defined by the oscillatory integral (in the $\eta$ variable)

$$
\begin{aligned}
c_{0}(x, \xi) & =\int e^{i\langle x-y, \eta-\xi\rangle} \rho(x, y ; \eta) d y d \eta \quad \text { with } \\
\rho(x, y ; \eta)= & \chi_{1}(x) \chi_{2}(y) \tau\left(|x-y|\langle x\rangle^{-1}\right) \int_{0}^{1} d t \int_{0}^{1} d s(u(s ; x, y))^{-1} \\
& \cdot i k \kappa(s)\left(\left\langle A(v(s ; x, y)) \nabla_{\xi}, \nabla_{\xi}\right\rangle a\right)\left((x+y) / 2 ; \eta_{t}\right) .
\end{aligned}
$$

Now we inset in (A.8) $\tau\left(|\eta-\xi|\langle\xi\rangle^{-1}\right)+1-\tau\left(|\eta-\xi|\langle\xi\rangle^{-1}\right)$ and split $c_{0}$ into $c_{1}+c_{2}$. In particular,

$$
\begin{aligned}
c_{2}(x, \xi) & =\int e^{i\langle x-y, \eta-\xi\rangle}\left(1-\tau\left(|\eta-\xi|\langle\xi\rangle^{-1}\right) \rho(x, y ; \eta) d y d \eta\right. \\
& =\int e^{i\langle x-y, \eta-\xi\rangle}\left(1-\tau\left(|\eta-\xi|\langle\xi\rangle^{-1}\right)\left(L_{\xi, \eta, D_{y}}^{*}\right)^{p}(\rho(x, y ; \eta)) d y d \eta\right.
\end{aligned}
$$

for all $p \in \mathbb{N}$. By direct computations, we see that $c_{2} \in \mathcal{S}(-\infty, g)$ and $c_{1} \in \mathcal{S}(m h, g)$. Since for any symbol $r$, there exists a symbol $s$ in the same class such that $\mathrm{Op} r=$ $s^{w}$, the equations (A.1), (A.3), (A.6), and (A.7), yield the desired result.

\section{Appendix B. FunCtional CALCULUS For PSEUdodifFERENTIAL OPERATORS.}

Here we present a result on the functional calculus for pseudodifferential operators associated to the metric $g$ in (2.12). This result is probably not new but we did not find a proof in the literature. It follows quite directly from arguments in [Bo1] (see also [Le]). However we sketch the proof for completeness. We use notions and results from Subsection 2.2.

Recall that, for $\rho \in \mathbb{R}$, we denote by $\mathcal{S}^{\rho}$ the set of smooth functions $\varphi$ on $\mathbb{R}$ such that $\sup _{t \in \mathbb{R}}\langle t\rangle^{k-\rho}\left|\partial_{t}^{k} \varphi(t)\right|<\infty$. If we take a real symbol $a \in \mathcal{S}(m, g)$, then the operator $a^{w}$ is self-adjoint on the domain $\mathcal{D}\left(a^{w}\right)=\left\{u \in \mathrm{L}^{2}\left(\mathbb{R}_{x}^{d}\right) ; a^{w} u \in \mathrm{L}^{2}\left(\mathbb{R}_{x}^{d}\right)\right\}$. In particular, the operator $\varphi\left(a^{w}\right)$ is well defined by the functional calculus if $\varphi$ is a borelean function on $\mathbb{R}$. We assume that $m \geq 1$. A real symbol $a \in \mathcal{S}(m, g)$ is said elliptic if $(i-a)^{-1}$ belongs to $\mathcal{S}\left(m^{-1}, g\right)$.

Theorem B.1. Let $m \geq 1$ and $a \in \mathcal{S}(m, g)$ be real and elliptic. Take $\varphi \in \mathcal{S}^{\rho}$. Then $\varphi(a) \in \mathcal{S}\left(m^{\rho}, g\right)$ and there is $b \in \mathcal{S}\left(h m^{\rho}, g\right)$ such that

$$
\varphi\left(a^{w}(x, D)\right)=(\varphi(a))^{w}(x, D)+b^{w}(x, D) .
$$


Proof. Let $\rho^{\prime} \in \mathbb{R}, \varphi \in \mathcal{S}^{\rho^{\prime}}$, and $k \in \mathbb{N}$ large enough such that $2 k>\rho^{\prime}$. Then $\psi(t):=\varphi(t)\left(1+t^{2}\right)^{-k}$ belongs to $\mathcal{S}^{\rho^{\prime}-2 k}$ with $\rho^{\prime}-2 k<0$. If the result is valid for $\rho<0$, then there exists $b \in \mathcal{S}\left(h m^{\rho^{\prime}-2 k}, g\right)$ such that

$$
\begin{aligned}
\varphi\left(a^{w}\right) & =\psi\left(a^{w}\right)\left(1+\left(a^{w}\right)^{2}\right)^{k}=\left((\psi(a))^{w}+b^{w}\right)\left(1+\left(a^{w}\right)^{2}\right)^{k} \\
& =\left(\psi(a)\left(1+a^{2}\right)^{k}\right)^{w}+c^{w}+\left(b \#\left(1+a^{2}\right)^{k}\right)^{w}=(\varphi(a))^{w}+d^{w}
\end{aligned}
$$

with $c, d \in \mathcal{S}\left(h m^{\rho^{\prime}}, g\right)$, by the composition properties. So it suffices to prove the result for $\rho<0$. Since we can write any function $\varphi \in \mathcal{S}^{\rho}$, with $\rho<0$, as $\varphi_{1} \varphi_{2}$ with $\varphi_{1} \in \mathcal{S}^{\delta}(-1 \leq \delta<0)$ and $\varphi_{1} \in \mathcal{S}^{[\rho]+1}$ (where $[\rho]$ denotes the integer part of $\rho$ ) and use the previous composition properties, we see by induction that it suffices to establish the result for $-1 \leq \rho<0$.

Let $z \in \mathbb{C} \backslash \mathbb{R}$. Using that $(z-a)^{-1}=(i-a)^{-1}\left(1+(z-i)(z-a)^{-1}\right)$, we observe that $\left|(z-a)^{-1}\right| \leq m^{-1}\langle z\rangle|\operatorname{Im}(z)|^{-1}$. Thus, for all $\ell \in \mathbb{N}$, there exists $C_{\ell}>0$ and $N_{\ell} \in \mathbb{N}$ such that, for all $z \in \mathbb{C} \backslash \mathbb{R},\left\|(z-a)^{-1}\right\|_{\ell, \mathcal{S}\left(m^{-1}, g\right)} \leq C_{\ell}\langle z\rangle^{N_{\ell}+1}|\operatorname{Im}(z)|^{-N_{\ell}-1}$. Define $q_{z}:=(z-a)^{-1} \#(z-a)-1 \in \mathcal{S}(h, g)$. By an explicit formula given in [Bo2] (first formula on page III-4), $q_{z}$ only depends on the derivatives of $(z-a)$, which are independent of $z$. Thus one can find, for all $\ell, C_{\ell}^{\prime}>0$ and $N_{\ell}^{\prime} \in \mathbb{N}$ such that

$$
\forall z \in \mathbb{C} \backslash \mathbb{R}, \quad\left\|q_{z}\right\|_{\ell, \mathcal{S}(h, g)} \leq C_{\ell}^{\prime}\langle z\rangle^{N_{\ell}^{\prime}+1}|\operatorname{Im}(z)|^{-N_{\ell}^{\prime}-1} .
$$

According to [Bo1], one can prove from the boundedness of commutators of $(z-$ $\left.a^{w}\right)^{-1}$ with appropriate pseudodifferential operators that this resolvent $\left(z-a^{w}\right)^{-1}$ is equal to $r_{z}^{w}$, where the symbol $r_{z}$ belongs to $\mathcal{S}\left(m^{-1}, g\right)$. Furthermore, the system $\|\cdot\|_{\ell, \mathcal{S}(m, g)}, \ell \in \mathbb{N}$, of semi-norms is equivalent to another one based on the previous commutators. Using this, there exist, for all $\ell, C_{\ell}^{\prime \prime}>0$ and $N_{\ell}^{\prime \prime} \in \mathbb{N}$ such that

$$
\forall z \in \mathbb{C} \backslash \mathbb{R}, \quad\left\|r_{z}\right\|_{\ell, \mathcal{S}\left(m^{-1}, g\right)} \leq C_{\ell}^{\prime \prime}\langle z\rangle^{N_{\ell}^{\prime \prime}+1}|\operatorname{Im}(z)|^{-N_{\ell}^{\prime \prime}-1} .
$$

Using (B.2) and (B.3), we can find, for all $\ell, C_{\ell}^{\prime \prime \prime}>0$ and $N_{\ell}^{\prime \prime \prime} \in \mathbb{N}$ such that

$$
\forall z \in \mathbb{C} \backslash \mathbb{R}, \quad\left\|q_{z} \# r_{z}\right\|_{\ell, \mathcal{S}\left(h m^{-1}, g\right)} \leq C_{\ell}^{\prime \prime \prime}\langle z\rangle^{N_{\ell}^{\prime \prime \prime}+1}|\operatorname{Im}(z)|^{-N_{\ell}^{\prime \prime}-1} .
$$

Now we take $\varphi \in \mathcal{S}^{\rho}$ with $-1 \leq \rho<0$ and consider some almost analytic extension $\varphi^{\mathbb{C}}$ (like in Proposition 2.4). Thanks to (B.4), (2.3), (2.4), and $\rho<0$,

$$
b:=\frac{i}{2 \pi} \int_{\mathbb{C}} \partial_{\bar{z}} \varphi^{\mathbb{C}}(z) q_{z} \# r_{z} d z \wedge d \bar{z}
$$

converges in $\mathcal{S}\left(h m^{-1} g\right)$. According to the definition of $q_{z},\left((z-a)^{-1}\right)^{w}\left(z-a^{w}\right)=$ $\operatorname{Id}+q_{z}^{w}$, thus $\left((z-a)^{-1}\right)^{w}=\left(z-a^{w}\right)^{-1}+\left(q_{z} \# r_{z}\right)^{w}$. Using Helffer-Sjöstrand formula $(2.6),(\varphi(a))^{w}=\varphi\left(a^{w}\right)+b^{w}$ with $b \in \mathcal{S}\left(h m^{-1} g\right) \subset \mathcal{S}\left(h m^{\rho} g\right)$, since $-1 \leq \rho$.

\section{Appendix C. An interpolation's ARgument.}

By pseudodifferential calculus, $A_{1}^{2}\left\langle D_{x}\right\rangle^{-2}\langle x\rangle^{-2}$ extends to a bounded operator on $\mathrm{L}^{2}\left(\mathbb{R}_{x}^{d}\right)$. What about $\left\langle A_{1}\right\rangle^{r}\left\langle D_{x}\right\rangle^{-r}\langle x\rangle^{-r}$ with $r>0$ ? The same argument is not clear since $A_{1}$ is not elliptic. Indeed its symbols $(x, \xi) \mapsto x \cdot \xi$ can vanish when $\xi \neq 0$. Using interpolation, we show

Lemma C.1. For real $r \geq 0,\left\langle A_{1}\right\rangle^{r}\left\langle D_{x}\right\rangle^{-r}\langle x\rangle^{-r}$ extends to a bounded operator on $\mathrm{L}^{2}\left(\mathbb{R}_{x}^{d}\right)$.

We refer to [MS][Lemma 7.1] for an alternative proof and historical remarks. 
Proof of Lemma C.1. We prove that, for $r \geq 0$,

$$
\exists C_{r}>0 ; \forall f \in \mathrm{L}^{2}\left(\mathbb{R}_{x}^{d}\right),\left\|\left\langle A_{1}\right\rangle^{r} f\right\| \leq C_{r}\left\|\langle x\rangle^{r}\left\langle D_{x}\right\rangle^{r} f\right\| .
$$

For $r \in \mathbb{N},\left(A_{1}+i\right)^{r}\left\langle D_{x}\right\rangle^{-r}\langle x\rangle^{-r}$ extends to a bounded operator by the pseudodifferential calculus with the metric $g$ in (2.12). Since $\left\langle A_{1}\right\rangle^{r}\left(A_{1}+i\right)^{-r}$ is bounded, (C.1) is satisfied when $r \in \mathbb{N}$. For $t, t^{\prime} \geq 0$, let $\mathrm{H}_{t}^{t^{\prime}}:=\left\{f \in \mathrm{L}^{2}\left(\mathbb{R}_{x}^{d}\right) ;\left\|\langle x\rangle^{t}\left\langle D_{x}\right\rangle^{t^{\prime}} f\right\|<\infty\right\}$. Now, using [Be], we infer that the space $\mathrm{H}_{r}^{r}$ is also the complex interpolated space $\left[\mathrm{H}_{0}^{0}, \mathrm{H}_{m}^{m}\right]_{r / m}$, where $m \geq r$. To be precise, use [Be] $[(1.7)]$ and notice that $H(m, g)=\mathrm{H}_{r}^{r}$, where $g$ is given as in (2.12) and $m(x, \xi)=\langle x\rangle^{r}\langle\xi\rangle^{r}$, by [Be][Theorem 3.7]. We deduce that (C.1) is true for all $r \geq 0$ by the Riesz-Thorin Theorem.

\section{Appendix D. A simpler argument in Dimension $d=1$.}

Here we present a more elementary proof of Lemma 5.5 in dimension $d=1$. It relies on the following

Lemma D.1. For $z \notin \mathbb{R}$, as bounded operators on $\mathrm{L}^{2}\left(\mathbb{R}_{x}\right)$,

$$
\left(z-D_{x}^{2}\right)^{-1} e^{ \pm i k x}=e^{ \pm i k x}\left(z-\left(D_{x} \pm k\right)^{2}\right)^{-1} .
$$

Proof. As differential operators, $D_{x} e^{ \pm i k x}=e^{ \pm i k x}\left(D_{x} \pm k\right)$. Thus, on $\mathrm{H}^{2}\left(\mathbb{R}_{x}\right)$, $\left(D_{x}^{2}-z\right) e^{ \pm i k x}=e^{ \pm i k x}\left(\left(D_{x} \pm k\right)^{2}-z\right)$. Multiplying on the left and on the right by the convenient resolvent, we get the result.

We first follow the general proof until formula (5.9). By (D.1),

$$
B_{1} \simeq-\sum_{\sigma= \pm} \frac{i q k}{4 \pi} \int_{\mathbb{C}} \partial_{\bar{z}} \varphi^{\mathbb{C}}(z)\left(z-D_{x}^{2}\right)^{-1}\left(z-\left(D_{x}+\sigma k\right)^{2}\right)^{-1} d z \wedge d \bar{z} e^{i \sigma k x} .
$$

Choosing the support of $\varphi$ small enough, we can find $\theta \in \mathcal{C}_{c}^{\infty}(\mathbb{R})$ such that $\xi \mapsto \theta\left(\xi^{2}\right)$ vanishes near $-k / 2$ and $k / 2, \varphi\left(\xi^{2}\right) \theta\left(\xi^{2}\right)=0=\varphi\left((\xi+k)^{2}\right) \theta\left(\xi^{2}\right)$, for all $\xi \in \mathbb{R}$, and such that the function $\xi \mapsto \varphi\left((\xi-k)^{2}\right) \theta\left(\xi^{2}\right)$ is nonzero (using that $\left.\mathcal{I} \subset\right] 0 ;+\infty[$ ). Set $B=\theta\left(D_{x}^{2}\right)$. Since $\xi^{2}-(\xi+k)^{2}$ and $\xi^{2}-(\xi-k)^{2}$ do not vanish on the support of $\theta\left(\xi^{2}\right)$,

$$
\begin{aligned}
B B_{1} \simeq-\sum_{\sigma= \pm} \frac{i q k}{4 \pi} \theta\left(D_{x}^{2}\right)\left(D_{x}^{2}-\left(D_{x}+k \sigma\right)^{2}\right)^{-1} \int_{\mathbb{C}} \partial_{\bar{z}} \varphi^{\mathbb{C}}(z)\left(z-D_{x}^{2}\right)^{-1} \\
\left(D_{x}^{2}-\left(D_{x}+k \sigma\right)^{2}\right)\left(z-\left(D_{x}+\sigma k\right)^{2}\right)^{-1} d z \wedge d \bar{z} e^{i \sigma k x} .
\end{aligned}
$$

By the resolvent formula and (2.6),

$$
B B_{1} \simeq-\sum_{\sigma= \pm} \frac{q k}{2} \theta\left(D_{x}^{2}\right)\left(D_{x}^{2}-\left(D_{x}+k \sigma\right)^{2}\right)^{-1}\left(\varphi\left(D_{x}^{2}\right)-\varphi\left(\left(D_{x}+k \sigma\right)^{2}\right)\right) e^{i \sigma k x} .
$$

Using the support properties of $\theta$, we obtain

$$
B B_{1} \simeq 2^{-1} q k \theta\left(D_{x}^{2}\right)\left(D_{x}^{2}-\left(D_{x}-k\right)^{2}\right)^{-1} \varphi\left(\left(D_{x}-k\right)^{2}\right) e^{-i k x} .
$$

Denoting by $B^{\prime}$ the multiplication operator by $e^{i k x}, B B_{1} B^{\prime}$ is, modulo some compact operator, a self-adjoint Fourier multiplier. The spectrum of the latter is given by the essential range of the function $\xi \mapsto 2^{-1} q k \theta\left(\xi^{2}\right)\left(\xi^{2}-(\xi-k)^{2}\right)^{-1} \varphi\left((\xi-k)^{2}\right)$. Since this function is non constant and continuous, the spectrum contains an interval and the corresponding operator cannot be compact. Thus $B B_{1} B^{\prime}$ is not compact. This finishes the proof of Lemma 5.5 in dimension $d=1$. 


\section{REFERENCES}

[Ag] S. Agmon: Lower bounds for solutions of Schrödinger equations. J. Analyse Math. 23, 1970, 1-25.

[ABG] W.O. Amrein, A. Boutet de Monvel, V. Georgescu: Co-groups, commutator methods and spectral theory of N-body hamiltonians., Birkhäuser 1996.

[BFS] V. Bach, J. Fröhlich, I.M. Sigal: Quantum electrodynamics of confined non-relativistic particles. Adv. in Math. 137, 299-395, 1998.

[Be] R. Beals: Weighted distribution spaces and pseudodifferential operators, J. Analyse Math. 39 (1981), 131-187.

[BD] M. Ben-Artzi, A. Devinatz: Spectral and scattering theory for the adiabatic oscillator and related potentials. J. Maths. Phys. 111 (1979), p. 594-607.

[BCHM] J-F. Bony, R. Carles, D. Häfner, L. Michel: Scattering theory for the Schrödinger equation with repulsive potential. J. Math. Pures Appl. 84, no. 5, 509-579, 2005.

[Bo1] J-M. Bony: Caractérisation des opérateurs pseudo-différentiels. Séminaire ÉDP (19961997), exposé numéro XXIII.

[Bo2] J-M. Bony: Sur l'ingalité de Fefferman-Phong. Séminaire ÉDP (1998-1999), exposé numéro III.

[BC] J-M. Bony, J-Y. Chemin: Espaces fonctionnels associés au calcul de Weyl-Hörmander. Bulletin de la S.M.F., tome 122, numéro 1 (1994), p. 77-118.

[BG] N. Boussaid, S. Golénia: Limiting absorption principle for some long range pertubations of Dirac systems at threshold energies. Comm. Math. Phys. 299, 677-708 (2010).

[Bu] N. Burq: Semiclassical estimates for the resolvent in non trapping geometries. Int. Math. Res. Notices 2002, no 5, 221-241.

[CJ] F. Castella, Th. Jecko: Besov estimates in the high-frequency Helmholtz equation, for a non-trapping and $C^{2}$ potential. J. Diff. Eq. Vol. 228, N.2, p. 440-485 (2006).

[Ca] L. Cattaneo: Mourre's inequality and Embedded bounded states, Bull. Sci Math. 129, Issue $7,591-614,2005$.

[CGH] L. Cattaneo, G. M. Graf, W. Hunziker: A general resonance theory based on Mourre's inequality, Ann. H. Poincaré 7 (2006), 3, p. 583-601.

[CHM] J. Cruz-Sampedro, I. Herbst, R. Martinez-Avendaño: Pertubations of the Wigner-Von Neumann potential leaving the embedded eigenvalue fixed. Ann. H. Poincaré 3 (2002) 331-345.

[CFKS] H.L. Cycon, R.G. Froese, W. Kirsch, B. Simon: Schrödinger operators with applications to quantum mechanics and global geometry. Springer Verlag 1987.

[DG] J. Dereziński, C. Gérard: Scattering theory of classical and quantum N-particle systems. Springer-Verlag 1997.

[DJ] J. Dereziński, V. Jakšić: Spectral theory of Pauli-Fierz operators. J. Funct. Anal. 180, no 2, p. 243-327, 2001.

[DMR] A. Devinatz, R. Moeckel, P. Rejto: A limiting absorption principle for Schrödinger operators with Von Neumann-Wigner potentials. Int. Eq. and Op. Theory, vol. 14 (1991).

[FMS1] J. Faupin, J.S. Møller, E. Skibsted: Regularity of bound states. arXiv:1006.5871.

[FMS2] J. Faupin, J.S. Møller, E. Skibsted: Second order perturbation theory for embedded eigenvalues. arXiv:1006.5869.

[FH] R.G. Froese, I. Herbst: Exponential bounds and absence of positive eigenvalues for $N$ body Schrödinger operators. Comm. Math. Phys. 87, 429-447 (1982).

[FHHH1] R.G. Froese, I. Herbst, M. Hoffmann-Ostenhof, T. Hoffmann-Ostenhof: $L^{2}$-Exponential Lower Bounds to solutions of the Schrödinger Equation. Comm. Math. Phys. 87, 265286 (1982).

[FHHH2] R.G. Froese, I. Herbst, M. Hoffmann-Ostenhof, T. Hoffmann-Ostenhof : On the absence of positive eigenvalues for one-body Schrödinger operators. J. Analyse Math. 41, 272284, (1982).

[GGé] V. Georgescu, C. Gérard: On the Virial Theorem in Quantum Mechanics, Comm. Math. Phys. 208, 275-281, (1999).

[GGM1] V. Georgescu, C. Gérard, J.S. Møller: Commutators, $C_{0}$-semigroups and resolvent estimates, J. Funct. Anal. 216, no 2, p. 303-361, 2004.

[GGo] V. Georgescu, S. Golénia: Isometries, Fock spaces and spectral analysis of Schrödinger operators on trees., J. Funct. Anal. 227 (2005), 389-429. 
[Gé] C. Gérard: A proof of the abstract limiting absorption principle by energy estimates, J. Funct. Anal. 254 (2008), no 11, 2707-2724.

[GJ] S. Golénia, Th. Jecko: A new look at Mourre's commutator theory, Compl. Ana. Op. Theory, Vol. 1, No. 3, p. 399-422, August 2007.

[GM] S. Golénia, S. Moroianu: Spectral analysis of magnetic Laplacians on conformally cusp manifolds., Ann. H. Poincaré 9 (2008), no. 1, 131-179.

[HeS] B. Helffer, J. Sjöstrand: Opérateurs de Schrödinger avec champs magnétiques faibles et constants. Exposé No. XII, Séminaire EDP, février 1989, Ecole Polytechnique.

[Hö1] L. Hörmander: The analysis of linear partial differential operators I., Springer-Verlag Berlin Heidelberg 1983.

[Hö2] L. Hörmander: The analysis of linear partial differential operators II., Springer-Verlag Berlin Heidelberg 1983.

[Hö3] L. Hörmander: The analysis of linear partial differential operators III., Springer-Verlag Berlin Heidelberg 1983.

[HuS] W. Hunziker, I.M. Sigal: The quantum N-body problem, J. Math. Phys. 41 (6), 34483510,2000 .

[IJ] A.D. Ionescu, D. Jerison: On the absence of positive eigenvalues of Schrödinger operators with rough potentials, GAFA, Geom. Funct. Anal. 13, 2003, 1029-1081.

[Je1] T. Jecko: From classical to semiclassical non-trapping behaviour, C. R. Acad. Sci. Paris, Ser. I, 338, p. 545-548, 2004.

[Je2] T. Jecko: Non-trapping condition for semiclassical Schrödinger operators with matrixvalued potentials. Math. Phys. Electronic Journal, No. 2, vol. 11, 2005.

[JMP] A. Jensen, E. Mourre, P. Perry: Multiple commutator estimates and resolvent smoothness in quantum scattering theory. Ann. Inst. H. Poincaré vol. 41, no 2, 1984, p. 207-225.

[Ka] T. Kato: Pertubation theory for linear operators. Springer-Verlag 1995.

[Ki] A. Kiselev: Imbedded singular continuous spectrum for Schrödinger operators, J. Amer. Math. Soc. 18 (2005), no. 3, 571603.

[Le] N. Lerner: Metrics on phase space and non-selfadjoint pseudodifferential operators. Birkhaüser 2010 .

[MU] K. Mochizuki, J. Uchiyama: Radiation conditions and spectral theory for 2-body Schrödinger operators with "oscillating" long-range potentials I: the principle of limiting absorption. J. Math. Kyoto Univ. 18, 2, 377-408, 1978.

$[\mathrm{M} \varnothing] \quad$ J. S. Møller: An abstract radiaton condition and applications to $N$-body systems., Reviews of Math. Phys., Vol. 12, No. 5 (2000), 767-803.

[MS] J.S. Møller, E. Skibsted: Spectral theory of time-periodic many-body systems. Adv. Math. 188 (2004), no. 1, 137-221.

[Mo] E. Mourre: Absence of singular continuous spectrum for certain self-adjoint operators. Comm. in Math. Phys. 78, 391-408, 1981.

[RS4] M. Reed, B. Simon: Methods of Modern Mathematical Physics, Tome IV: Analysis of operators. Academic Press.

[ReT1] P. Rejto, M. Taboada: A limiting absorption principle for Schrödinger operators with generalized Von Neumann-Wigner potentials I. Construction of approximate phase. J. Math. Anal. and Appl. 208, p. 85-108 (1997).

[ReT2] P. Rejto, M. Taboada: A limiting absorption principle for Schrödinger operators with generalized Von Neumann-Wigner potentials II. The proof. J. Math. Anal. and Appl. 208, p. 311-336 (1997).

[Re] C. Remling: The absolutely continuous spectrum of one-dimensional Schrödinger operators with decaying potentials, Comm. Math. Phys. 193 (1998), 151-170.

[RoT] D. Robert, H. Tamura: Semiclassical estimates for resolvents and asymptotics for total cross-section. Ann. Inst. H. Poincaré 46, 415-442 (1987).

[Roy] J. Royer: Limiting absorption principle for the dissipative Helmholtz equation. CPDE 35, 1458-1489 (2010).

[Sa] J. Sahbani: The conjugate operator method for locally regular Hamiltonians. J. Oper. Theory 38, No. 2, 297-322 (1997).

[Si] B. Simon: On positive eigenvalues of one body Schrödinger operators. Comm. Pure Appl. Math. 22, 531-538 (1969).

[Wa] X.P. Wang: Semiclassical resolvent estimates for $N$-body Schrödinger operators. J. Funct. Anal. 97, 466-483 (1991). 
GOLÉNIA, SYLVAIN AND JECKO, THIERRY

[WZ] X.P. Wang, P. Zhang: High frequency limit of the Helmholtz equation with variable refraction index. J. Funct. Anal. 230, 116-168 (2006).

imB, UMR 5251 du CNRS, Université de Bordeaux 1, 351 Cours de la Libération, F-33 405 Talence Cedex, France

E-mail address: Sylvain.Golenia@math.u-bordeaux1.fr

AGM, UMR 8088 du CNRS, Université de Cergy Pontoise, Site de Saint Martin, 2, rue Adolphe Chauvin, F-95000 Cergy-Pontoise, France

E-mail address: thierry.jecko@u-cergy.fr 